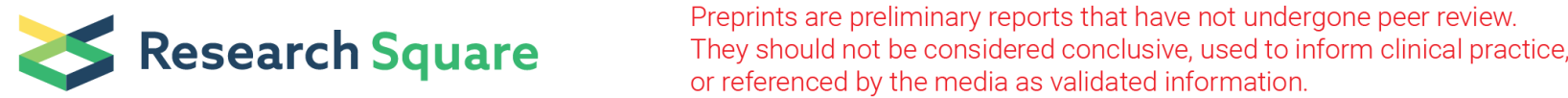

\section{Tip Trajectory Characteristics and Nonlinear Stability Analysis of Robotic Manipulator With Flexible Links-joints}

\section{Pravesh Kumar}

Indian Institute of Technology Rajasthan: Indian Institute of Technology Jodhpur

Barun Pratiher ( $\nabla$ barun@iitj.ac.in )

Indian Institute of Technology Rajasthan: Indian Institute of Technology Jodhpur

https://orcid.org/0000-0002-1219-6923

\section{Research Article}

Keywords: Two-link manipulator, link and joint flexibility, prismatic-revolute joints, modal analysis, dynamic responses, bifurcation \& stability

Posted Date: July 9th, 2021

DOI: https://doi.org/10.21203/rs.3.rs-603725/v1

License: (c) (i) This work is licensed under a Creative Commons Attribution 4.0 International License. Read Full License 


\title{
Tip trajectory characteristics and nonlinear stability analysis of robotic manipulator with flexible links-joints
}

\author{
Pravesh Kumar and Barun Pratiher* \\ Department of Mechanical Engineering, Indian Institute of Technology Jodhpur, India-342037 \\ *Corresponding Author's email id: barun@iitj.ac.in
}

An efficient and new dynamic model of two-link flexible manipulator connected and controlled by flexible joints i.e., prismatic and revolute pairs has been developed to explore the modal analysis to study tip-trajectory characteristics and subsequently investigate the nonlinear steady-state responses under harmonic motion at flexible joints. The governing equations including joint dynamics have been derived using extended Hamilton's principle. Modal parameters have been graphically presented to highlight the influences of various system parameters on the determination of eigenfrequencies and eigenspectrums. Obtained reduced order equations have then used to study the trajectory characteristics of tip displacements, angular and actuator positions by imparting the appropriate actuator force and joint torque. Further, nonlinear studies have been carried out to compute the steady state responses and their stability and local bifurcation by using $2^{\text {nd }}$ order method of multiple scales. Investigation of the influences of various design parameters on the nonlinear stability and local bifurcation of steady state responses have been demonstrated and those results have been found to be in good agreement with numerically obtained findings. The obtained results find very useful in the applications of long-reach robot manipulators performing complex operations assigning with translating and rotary motion together.

Keywords: Two-link manipulator; link and joint flexibility; prismatic-revolute joints; modal analysis; dynamic responses; bifurcation \& stability. 


\section{Introduction}

The modeling and subsequent control problems in flexible robotic system has been a major center of attention in recent years on the grounds that robots are being widely used in modern industrial operations, medical utilities, agricultural production, defense tasks and space exploration. In order to perform hazardous operations, where human reach is not possible, for example in nuclear industry, long reach robotic manipulators are being used effectively. In medical industry, robots are being employed to accomplish tedious and intricate surgical tasks where the accuracy is of main concern. There has been a constant pursuit to have low power consumption, high speed, and light-weight manipulator in industrial and space applications in order to boost the productivity or reduce the payload of spacecraft. In pick and drop operation, the manipulator is supposed to move on a planar surface to reach the required location and then perform the requisite tasks. Flexible robot manipulator with a prismatic joint are functioning in many such engineering applications like industrial robots, telescopic members attached to loading vehicles, spacecraft antenna, magnetic tape-drivers, printers, band saws, and weaving mechanisms. Hence, developing at least a two-link flexible manipulator with harmonically driven prismatic and revolute joints incorporating required payload can have useful engagement in various industrial operations, especially space applications where a long-reach and lightweight robotic manipulator is highly necessitate. The flexibilities in the links, actuator as well as the joints have been introduced in resemblance with the actual manipulator models. Numerous studies have been carried out with regard to the investigation of modeling, and dynamic control of single-link manipulator having revolute joint, with and without payload. The reported studies have been divided into two categories based on the type of joints used to drive the manipulator i.e. revolute [1-5] and prismatic joints [6-11]. On many occasions, beam element is often used to model the flexible link and integrating it with rotating hub makes a robotic manipulator with revolute joint [12]. Fundamental beam element has been often used to model one-dimensional flexible link in vibration analysis of flexible robots [13]. The linear and nonlinear dynamic characteristics of these one-dimensional flexible links have been investigated using either classical or refined beam theories (Timoshenko or higher order shear beam). However, EulerBernoulli beam hypothesis [14], has been one of the most elementary beam element theory widely used to estimate the transverse deflection and eigenfrequencies of flexible robots arms. It is very evident from the existing literature that considering appropriate beam theory to suitably model a flexible robotic arm always leads to accuracy in evaluating the desired dynamic responses when subjected to external influences. Appropriate identification of tip mass attached at tip of manipulator link [15] that can often assumed as payload or end-effector of the robotic manipulator not only affects vibration frequencies but also changes the joint requirements as torques or forces for a specific maneuver in a pick and place operation. The dynamics of longreach manipulator link with end-effector has mostly been modeled using Euler-Bernoulli's beam with attached tip mass. The dynamics of beam deformation, link flexibility results coupled nonlinear partial differential equation with complex boundary conditions. Linear analysis [16-18] is sometimes enough to describe the small vibration oscillation of flexible robotic link. Mostly, linear analysis has been restricted to calculate the eigenfrequencies and corresponding eigenfunctions. However, nonlinear analysis [18-20] is very imperative to compute the vibration behaviors of a flexible robotic manipulator under external and/or parametric influences as the system exhibits inadmissible vibrations when the forcing frequency becomes equal or nearly 
equal to its natural frequency. Various types of nonlinearities exist in a manipulator system such as inertial, geometric, damping, material and physical nonlinearities. While inertial nonlinearities $[5,18]$ generally occur due the nonlinear terms in the velocities of the links, geometric nonlinearity $[6,19,20]$ arises in the potential/strain energy terms of manipulator for inextensible, large deformation or nonlinear curvature conditions of the links that result into cubic nonlinear terms in the equations of the motion. Often the nonlinear stress-strain relationship [6] due to inelastic deformation in the link enables substantial effect on the structural responses under forcing conditions. The dynamics of the long-reach [21] or flexible manipulator are significantly complex by the virtue of flexible nature of the system. As a result, these flexibilities in the manipulator links and joints possess a major challenge to achieve and maintain accurate positioning and desired trajectory tracking. As the robot manipulator in various capacities and configurations are being used in different industrial operations for different applications where the end point control is of major concern, it becomes inevitable to study the system performance under parametric variations. However, the rich and diverse dynamics of flexible robots have been discretized using various solution techniques such as assumed mode method (AMM) [22-24], finite element method (FEM) [25-27], and lumped parameter method (LPM) [28-29] to study its behavior under different working conditions.

The suitable understanding of the eigenfrequencies $[12,16,18]$ and vibration characteristics of flexible manipulator enhances the ability of a design engineer to detect, locate and quantify the extent of damages within the robotic structure. The fundamental frequency may vary significantly with the design parameters which in turn possesses a challenge to the robustness of a designed controller. Identifying the eigenfrequencies and corresponding mode shapes may offer necessary information about the deflected shape of structural vibration to design engineer when one of the natural frequencies comes closer to the external frequency called as resonance. The effect of design parameters on the system's natural frequencies and subsequent mode shapes before moving into the investigation of the dynamic performance of flexible manipulator is substantial requirements. Investigation of parametric variations on modal analysis can offer the ways of improving and optimizing the dynamic aspects of flexible manipulators. Proper knowledge about modal parameters always guides the operator to appropriately select the desired operating conditions in order to avoid any resonance condition that causes the unwanted excessive vibration and noise.

Furthermore, flexible robots possess higher elastic compliance that leads to residual vibrations especially after a grasping maneuver. These light and flexible robot arms combined with fast motions or interacting with surrounding atmosphere or by stopping abruptly may cause considerable amount of vibration of the end point. This residual vibration may lead to positioning inaccuracy [33]. However, dynamics of robotic manipulator becomes more complex while considering both revolute and prismatic joints in addition to elastic compliance of flexible links. Flexible manipulator with both revolute and prismatic pairs raises the substantial difficulties in developing the mathematical model over the robotic system only with revolute joints [5, 14]. The earlier works lacked in the nonlinear behavior of the two-link flexible manipulator with prismatic and revolute pairs and thus major focus here is to investigate the nonlinear dynamic responses along with parametric study on the determination of its modal parameters. Determining the nonlinear responses [6, 18-20] enables a deep insight and better understanding of dynamic performances of a long-reach two-link manipulator to identify the ranges of operation safety and thus avoiding undesirable behavior because of structural instabilities [18-20]. Simultaneously, catastrophic failure under various configurations can be 
avoided by the sufficient knowledge of the system's behavior operating at a certain speed. Hence, investigation of nonlinear responses of flexible long-reach manipulator is quite necessary for accurately predicting the state of dynamic stability to avoid catastrophic failure under different working circumstances.

Based on the existing literature, it is quite apparent that the single-link and two-link manipulators with revolute joints have been modeled and studied thoroughly. Subsequent various control strategies [30-32] have been developed for vibration suppression in such manipulators. In addition, researchers $[6,18,19]$ have extensively investigated the resonant responses of singlelink flexible manipulator with prismatic joint under different working excitations. However, twolink manipulators considering prismatic joint has been constantly eluded from the research. As a result, there have been lacks of studies on dynamic responses of flexible manipulator with harmonically excited prismatic and revolute joints. Consequently, in this work, the dynamic model of two-link manipulator incorporating a payload driven by prismatic and revolute motions has been attempted. The governing equations of motion and boundary conditions assimilating geometric nonlinearities have been derived using extended Hamilton's principle with subsequent modal analysis to determine the eigenfrequencies and eigenfunctions. Due to behavioral uncertainty at payload end, accurate dynamic modeling of flexible manipulator with prismatic and revolute pairs possesses a challenging task and hence, investigation of influences of critical design parameters on the system responses can highlight its parametric instability under certain working situations. In nonlinear analysis, perturbation technique has been used to determine resonant responses under different conditions. Effect of design parameters such as payload mass, joint variables, mass density and flexural rigidity of the links onto the modal parameters, nonlinear behaviors and stability of the system has been exhibited graphically. The conferred results shall be noteworthy contribution in development of effective control strategies for vibration suppression of long reach manipulators.

\section{Flexible Two-link manipulator system: Kinematic model}

A schematic diagram of two-link planar flexible manipulator incorporating a payload is shown in Fig. 1. The first link of the manipulator is subjected to time dependent vertical motion $(\vartheta)$ at the actuator having mass $\left(\mathrm{m}_{\mathrm{a}}\right)$ and linear stiffness $\left(\mathrm{k}_{\mathrm{a}}\right)$ representing the prismatic joint while $\mathrm{v}_{\mathrm{a}}$ is the vertical displacement of actuator from the equilibrium position. The second link has been driven by revolute joint having mass $\left(\mathrm{m}_{1}\right)$, inertia $(\mathrm{J})$ and torsional spring stiffness $\left(\mathrm{k}_{\theta}\right)$ at one end and payload of mass $\left(\mathrm{m}_{2}\right)$ at other end. $(\mathrm{X}, \mathrm{Y})$ with $\left(\boldsymbol{K}, \mathbf{Y}^{\boldsymbol{t}}\right)$ represents the inertial Cartesian frame of co-ordinate systems fixed in space and $\left(\eta_{1}, \xi_{1}\right),\left(\eta_{2}, \xi_{2}\right)$ are the moving or local coordinate systems attached with first and second link, respectively. In the development of dynamic model of the two-link flexible manipulator assumptions such as 1) link deformation are small as compared to the length of the link; 2) tip angle $(\phi)$ is expressed in terms of end point transverse deflection $\left(\mathrm{s}_{\mathrm{L}}\right)$ of link and can assume as $\left.\phi=\tan ^{-1}\left(\mathrm{~s}_{\mathrm{L}}^{\prime}\right)\right) \approx \mathrm{s}_{\mathrm{L}}^{\prime}$; 3) the links behave as an Euler-Bernoulli beam element; 4) prismatic and revolute joints are modeled as linear springmass system, torsional spring-inertia system, respectively are taken into consideration. The expressions for the total kinetic energy ( $\mathrm{T}$ ) and potential energy ( $\mathrm{U}$ ) of the system, respectively are given by 


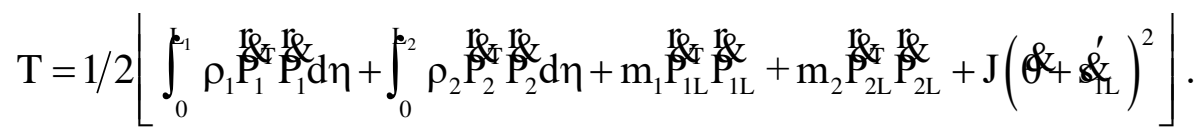

$$
\begin{aligned}
& \mathrm{U}=1 / 2\left(\int_{0}^{k_{1}} \mathrm{E}_{1} \mathrm{I}_{1}\left(\mathrm{~s}_{1}^{\prime}\right)^{\prime 2} \mathrm{~d} \eta+\int_{0}^{k_{2}} \mathrm{E}_{2} \mathrm{I}_{2}\left(\mathrm{~s}_{2}^{\prime}\right)^{2} \mathrm{~d} \eta\right)+ \\
& 1 / 2\left(\int_{0}^{k_{1}} E_{1} A_{1}\left((1 / 2) s_{1}^{\prime}{ }^{2}\right)^{2} d \eta+\int_{0}^{k_{2}} E_{2} A_{2}\left((1 / 2) s_{2}^{\prime 2}\right)^{2} d \eta\right)+ \\
& 1 / 2\left(\mathrm{k}_{\mathrm{a}}\left\{\mathrm{s}_{(\mathrm{x}=0)}+\vartheta(\mathrm{t})+\mathrm{v}_{\mathrm{a}}(\mathrm{t})\right\}^{2}+\mathrm{k}_{\theta}\left(\theta+\mathrm{s}_{1 \mathrm{~L}}^{\prime}\right)^{2}\right) .
\end{aligned}
$$

Here, $\mathrm{T}$ constitutes the kinetic energy associated with masses of the links $\left(\rho_{1,2}\right)$, masses at the link terminals $\left(m_{1,2}\right)$ and joint inertia $(j)$ while $U$ represents the bending strain energy of links ( $\left.E I_{=1,2}\right)$, energy due to axial stretching $\left(E A_{-1,2}\right)$, strain energy of the prismatic and revolute joints ( $\left.k_{a}, k_{\theta}\right)$. Subscripts 1,2 designate the first and second link respectively.

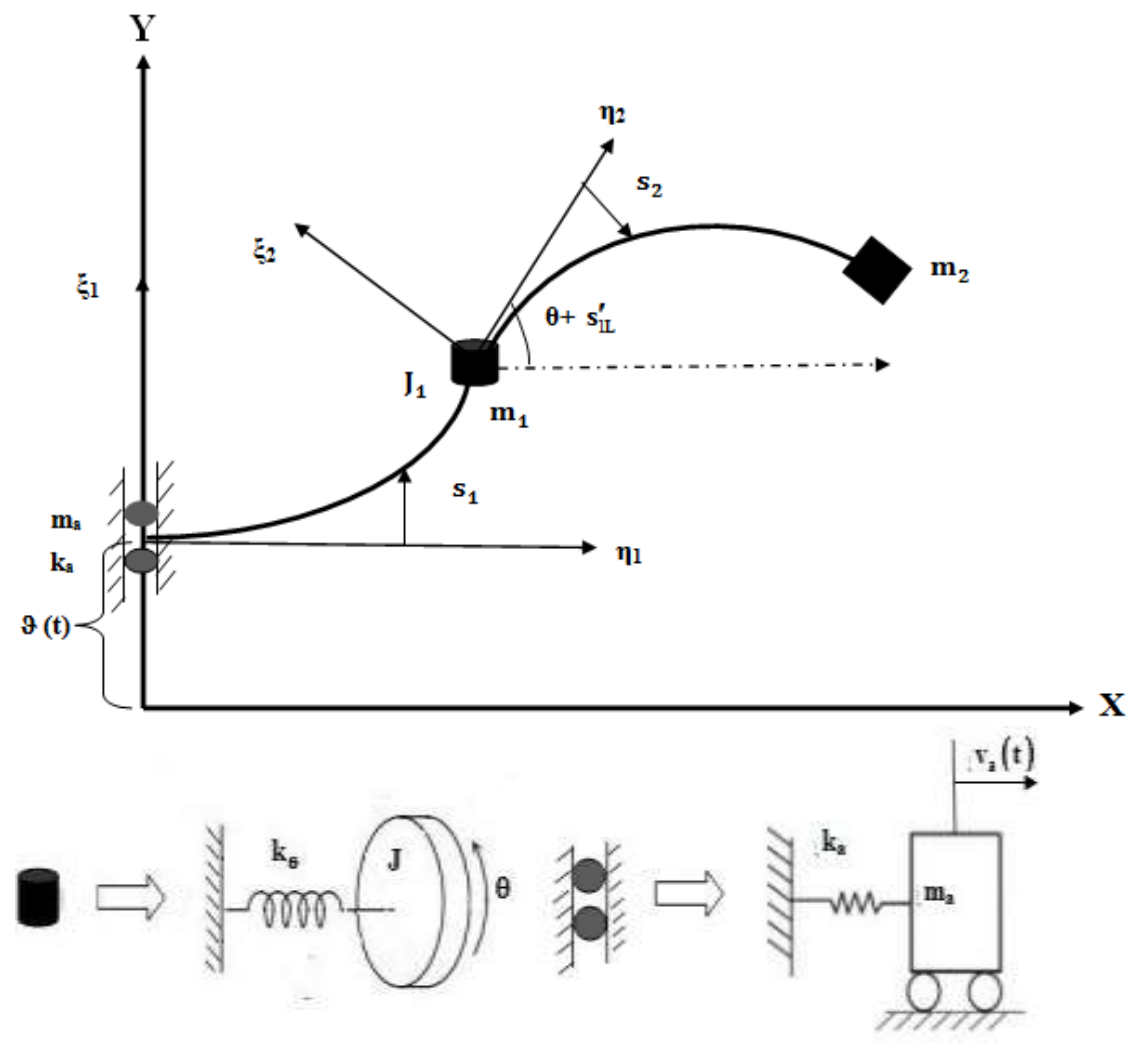

Fig. 1: Schematic diagram of a planar two-link flexible robotic manipulator with prismatic and intermediatory revolute joint incorporating a payload.

The mathematical expressions of the position vectors for the general point $(\mathrm{P})$ on the link and at end point $\left(\mathrm{P}_{\mathrm{L}}\right)$, respectively are:

$$
\stackrel{\mathrm{r}}{\mathrm{P}_{1}}=\left[\begin{array}{c}
\eta \\
(\mathrm{s}+\xi)
\end{array}\right]^{\left(\eta_{1}, \xi_{1}\right)}+\left[\begin{array}{c}
0 \\
\left(\mathrm{v}_{\mathrm{a}}+\vartheta\right)
\end{array}\right]^{(\mathrm{X}, \mathrm{Y})}, \stackrel{\mathrm{r}}{\mathrm{P}_{1 L}}=\left[\begin{array}{c}
\mathrm{L}_{1} \\
\mathrm{~s}_{1 \mathrm{~L}}
\end{array}\right]^{\left(\eta_{1}, \xi_{1}\right)}+\left[\begin{array}{c}
0 \\
\left(\mathrm{v}_{\mathrm{a}}+\vartheta\right)
\end{array}\right]^{(\mathrm{X}, \mathrm{Y})},
$$


$\stackrel{\mathrm{r}}{\mathrm{P}_{2}}=\stackrel{\mathrm{r}}{\mathrm{P}}+\left[\begin{array}{c}\eta \\ \left(\mathrm{s}_{2}+\xi\right)\end{array}\right]^{\left(\mathrm{r}_{2}, \xi_{2}\right)}+\left[\begin{array}{c}0 \\ \left(\mathrm{v}_{\mathrm{a}}+\vartheta\right)\end{array}\right]^{(\mathrm{X}, \mathrm{Y})}$ and $\stackrel{\mathrm{r}}{\mathrm{P}_{2 L}}=\stackrel{\mathrm{r}}{\mathrm{P}_{1}}+\left[\begin{array}{c}\eta \\ \mathrm{s}_{2 \mathrm{~L}}\end{array}\right]^{\left(\eta_{2}, \xi_{2}\right)}+\left[\begin{array}{c}0 \\ \left(\mathrm{v}_{\mathrm{a}}+\vartheta\right)\end{array}\right]^{(\mathrm{X}, \mathrm{Y})}$

Here, $(\eta, \xi)$ denotes the undeformed position of an arbitrary point on the link. The velocity vectors of the general point $\left(\begin{array}{l}\mathrm{P}_{1} \\ \mathrm{r}_{2}\end{array}\right)$ and end point of the links $\left(\mathrm{P}_{\mathrm{L}}^{\mathrm{r}}\right)$ can be obtained by differentiating with time as explained in Kumar and Pratiher [11].

\section{1: System Modeling: dynamic formulation}

The governing equations of link motions along with joint dynamics has been obtained for the two-link flexible manipulator by employing extended Hamilton's principle: $\sum_{1}^{2} \int_{\mathrm{t}_{1}}^{\mathrm{t}_{2}} \delta(\mathrm{T}-\mathrm{U}) \mathrm{dt}=0$, with $\delta\left(\mathrm{t}_{1}\right)=\delta\left(\mathrm{t}_{2}\right)$ equal to zero between two times stages. Now, by substituting equations (1-2) in extended Hamilton's principle, fourth-order coupled partial differential equations for the link motions and complex boundary conditions along with the joint dynamics are expressed as:

The governing equation of first link motion is expressed as:

$\rho_{1} \mathrm{~A}_{1}+\mathrm{E}_{1} \mathrm{I}_{1} \mathrm{~s}_{1}^{\prime \prime \prime}-(3 / 2) \mathrm{E}_{1} \mathrm{~A}_{1} \mathrm{~s}_{1}^{\prime 2} \mathrm{~s}_{1}^{\prime \prime}=0$.

The associated boundary conditions for the first link are:

$$
\begin{aligned}
& \left\{\mathrm{E}_{1} \mathrm{I}_{1} \mathrm{~s}_{1}^{\prime \prime \prime}+\mathrm{m}_{\mathrm{a}}\left(\mathrm{a}_{\mathrm{a}}\right)\right\}_{(0, \mathrm{t})}+\mathrm{k}_{\mathrm{a}}\left(\mathrm{s}_{1}+\vartheta+\mathrm{v}_{\mathrm{a}}\right)_{(0, \mathrm{t})}=0,\left\{\mathrm{E}_{1} \mathrm{I}_{1} \mathrm{~s}_{1}^{\prime}\right\}_{(0, \mathrm{t})}=0 \text {, }
\end{aligned}
$$

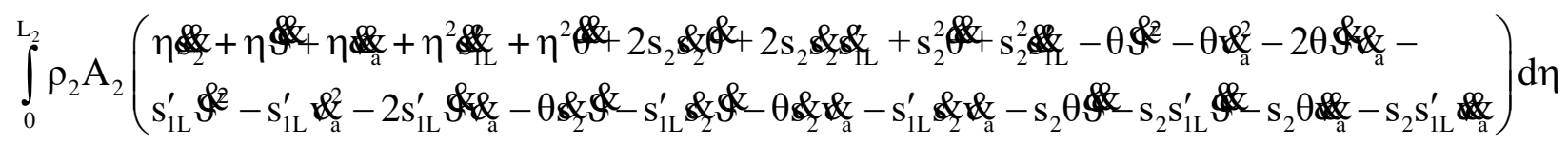

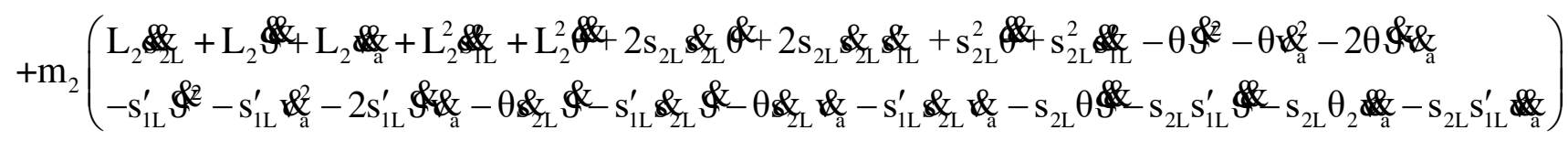

$$
\begin{aligned}
& +\mathrm{E}_{1} \mathrm{I}_{1} \mathrm{~s}_{1 \mathrm{~L}}^{\prime \prime}+\mathrm{J}(\mathrm{L})+\mathrm{k}_{\theta}\left(\theta+\mathrm{s}_{1 \mathrm{~L}}^{\prime}\right)-\mathrm{E}_{2} \mathrm{I}_{2} \mathrm{~s}_{2}{ }^{\prime \prime}(0, \mathrm{t})=0
\end{aligned}
$$

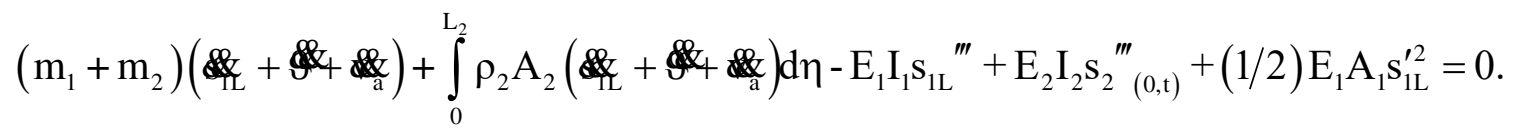

Similarly, the governing equation for second link motion can be expressed as:

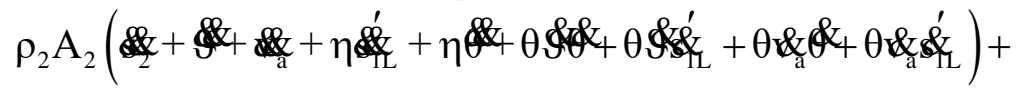

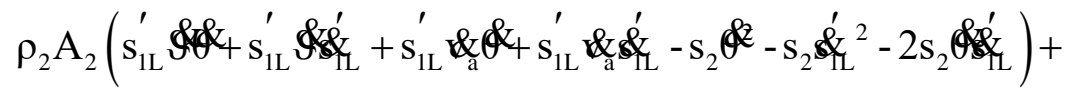

$\mathrm{E}_{2} \mathrm{I}_{2} \mathrm{~s}_{2}^{\prime \prime}-(3 / 2) \mathrm{E}_{2} \mathrm{~A}_{2} \mathrm{~s}_{2}^{\prime}{ }^{\prime} \mathrm{s}_{2}^{\prime \prime}=0$.

The associated boundary conditions for the second link are:

$$
\left\{\mathrm{E}_{2} \mathrm{I}_{2} \mathrm{~s}_{2}\right\}_{(0, \mathrm{t})}=\mathrm{E}_{1} \mathrm{I}_{1} \mathrm{~s}_{1 \mathrm{~L}},\left\{\mathrm{E}_{2} \mathrm{I}_{2} \mathrm{~s}_{2}^{\prime}\right\}_{(\mathrm{x}=0)}=\mathrm{E}_{1} \mathrm{I}_{1} \mathrm{~s}_{1 \mathrm{~L}}^{\prime},\left\{\mathrm{E}_{2} \mathrm{I}_{2} \mathrm{~s}_{2}^{\prime \prime}\right\}_{\left(\mathrm{L}_{2}, \mathrm{t}\right)}=0 \text {, }
$$




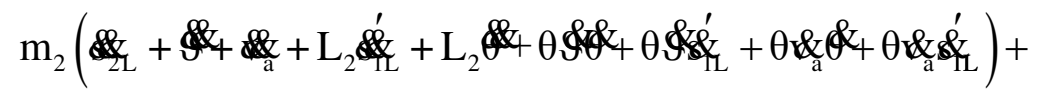

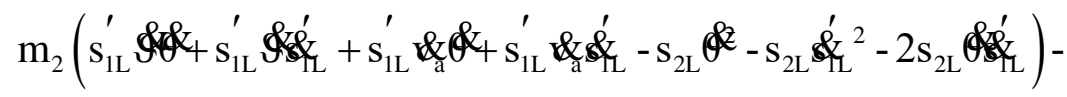

$\mathrm{E}_{2} \mathrm{I}_{2} \mathrm{~s}_{2 \mathrm{~L}}{ }^{\prime \prime}+(1 / 2) \mathrm{E}_{2} \mathrm{~A}_{2} \mathrm{~s}_{2 \mathrm{~L}}{ }^{3}=0$.

Equation of motion of joint dynamics is expressed as:

$\mathrm{J}\left(\mathrm{k}_{\theta}\left(\theta+\mathrm{s}_{\mathrm{lL}}^{\prime}\right)+\right.$

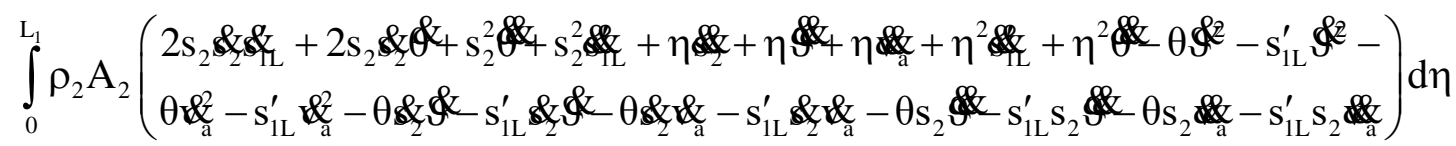

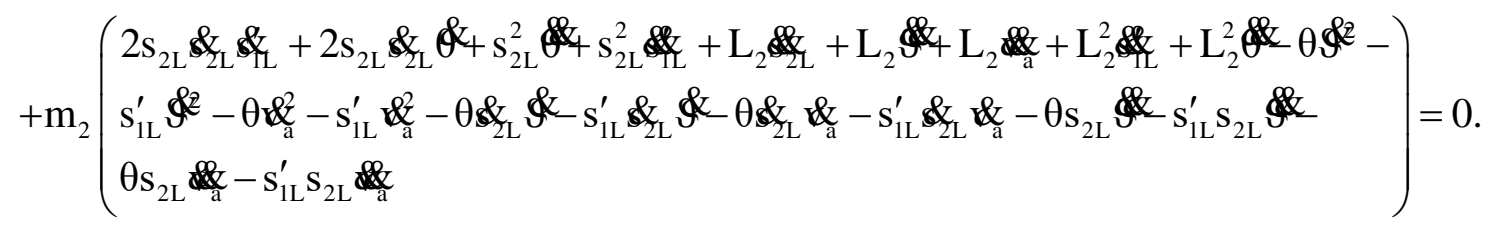

\subsection{Free Vibration: eigenfunction and eigenfrequencies}

In this section, the natural frequencies and related mode shapes are sought for the dynamic model using free vibration analysis. The effect of coupled nonlinear terms is then neglected and the transverse deflections of first and second links are expressed in terms of new functions in space and time as $n_{1}(\eta, t)=s_{1}(\eta, t)+\vartheta(t)+v_{a}(t)$ and $n_{2}(\eta, t)=s_{2}(\eta, t)+\vartheta(t)+v_{a}(t)+\eta\left(\theta+s_{1 L}^{\prime}\right)$ which on substitution in Eqs. (3-7) results into following equations given by:

$\rho_{1} \mathrm{~A}_{1}+\mathrm{E}_{1} \mathrm{I}_{1} \mathrm{n}_{1}^{\prime \prime \prime \prime}=0$.

$\left\{\mathrm{E}_{1} \mathrm{I}_{1} \mathrm{n}_{1}^{\prime \prime \prime}+\mathrm{m}_{\mathrm{a}} \mathrm{k}_{\mathrm{a}} \mathrm{n}\right\}_{(0, \mathrm{t})}=0,\left\{\mathrm{E}_{1} \mathrm{I}_{1} \mathrm{p}_{1}^{\prime}\right\}_{(0, \mathrm{t})}=0$,

$\int_{0}^{\mathrm{L}_{2}} \rho_{2} \mathrm{~A}_{2} \eta \mathrm{d} \eta+\mathrm{E}_{1} \mathrm{I}_{1} \mathrm{n}_{1 \mathrm{~L}}^{\prime \prime}+\mathrm{m}_{2} \mathrm{~L}_{2}+\mathrm{J}\left(\mathrm{k}_{\theta}\left(\mathrm{n}_{1 \mathrm{~L}}^{\prime}+\theta\right)-\left\{\mathrm{E}_{2} \mathrm{I}_{2} \mathrm{n}_{2}^{\prime \prime}\right\}_{(0, \mathrm{t})}=0\right.$

$\left(m_{1}+m_{2}+\rho_{2} A_{2} L_{2}\right)-E_{1} I_{1} n_{1 L}^{\prime \prime \prime}+\left\{E_{2} I_{2} n_{2}^{\prime \prime \prime}\right\}_{(0, t)}=0$.

$\rho_{2} \mathrm{~A}_{2}+\mathrm{E}_{2} \mathrm{I}_{2} \mathrm{n}_{2}^{\prime \prime \prime}=0$.

$\left\{\mathrm{E}_{2} \mathrm{I}_{2} \mathrm{n}_{2}\right\}_{(0, \mathrm{t})}=\mathrm{E}_{1} \mathrm{I}_{1} \mathrm{n}_{1 \mathrm{~L}}$

$\mathrm{E}_{2} \mathrm{I}_{2}\left\{\mathrm{n}_{2}^{\prime}-\theta-\mathrm{n}_{1 \mathrm{~L}}^{\prime}\right\}_{(0, \mathrm{t})}=\mathrm{E}_{1} \mathrm{I}_{1} \mathrm{n}_{1 \mathrm{~L}}, \mathrm{E}_{2} \mathrm{I}_{2} \mathrm{n}_{2 \mathrm{~L}}^{\prime \prime}=0, \mathrm{~m}_{2} \mathrm{n}_{2 \mathrm{~L}}^{\prime \prime}-\mathrm{E}_{2} \mathrm{I}_{2} \mathrm{n}_{2 \mathrm{~L}}^{\prime \prime \prime}=0$.

$\int_{0}^{L_{1}} \rho_{2} A_{2} \eta d \eta+m_{2} L_{2}+J\left(k_{\theta}\left(\theta+n_{1 L}^{\prime}\right)=0\right.$.

Now, the deflection functions $\left(\mathrm{n}_{1}, \mathrm{n}_{2}\right)$ are expressed as harmonic functions in terms of corresponding eigenfunctions $\{\mathrm{N}(\mathrm{x})\}$ and eigenfrequencies $(\omega)$ as $\mathrm{n}(\mathrm{x}, \mathrm{t})=\mathrm{N}(\mathrm{x}) \cos (\omega \mathrm{t})$. Also, the joint angular rotation $(\theta)$ is denoted as $\theta=\theta_{0} \cos (\omega t)$. Here, $N_{1}(x)$ and $N_{2}(x)$ are the corresponding eigenfunction for first and second link, respectively, $\omega$ is the unknown eigenfrequency of the whole system, while $\theta_{0}$ is the amplitude of joint rotation. 
After substituting of above mentioned deflection functions in the governing equations of motion, Eqs. (8 \& 10) and applying second boundary condition of first link, the respective eigenfunctions of first and second link, for the $\mathrm{m}^{\text {th }}$ mode of vibration, in terms of nondimensional space parameter $(\bar{\eta})$ and nondimensional eigenfrequency parameter $(\bar{\lambda})$, are expressed as:

$$
\begin{aligned}
& \mathrm{N}_{1}^{\mathrm{m}}(\bar{\eta})=\mathrm{R}_{1}^{\mathrm{m}} \mathrm{c}\left(\bar{\lambda}^{\mathrm{m}} \bar{\eta}\right)+\mathrm{R}_{2}^{\mathrm{m}}\left\{\mathrm{s}\left(\bar{\lambda}^{\mathrm{m}} \bar{\eta}\right)-\operatorname{sh}\left(\bar{\lambda}^{\mathrm{m}} \bar{\eta}\right)\right\}+\mathrm{R}_{3}^{\mathrm{m}} \operatorname{ch}\left(\bar{\lambda}^{\mathrm{m}} \bar{\eta}\right) \\
& \mathrm{N}_{2}^{\mathrm{m}}(\bar{\eta})=\mathrm{S}_{1}^{\mathrm{m}} \mathrm{c}\left(\kappa \bar{\lambda}^{\mathrm{m}} \bar{\eta}\right)+\mathrm{S}_{2}^{\mathrm{m}} \mathrm{s}\left(\kappa \bar{\lambda}^{\mathrm{m}} \bar{\eta}\right)+\mathrm{S}_{3}^{\mathrm{m}} \mathrm{ch}\left(\kappa \bar{\lambda}^{\mathrm{m}} \bar{\eta}\right)+\mathrm{S}_{4}^{\mathrm{m}} \operatorname{sh}\left(\kappa \bar{\lambda}^{\mathrm{m}} \bar{\eta}\right)
\end{aligned}
$$

Here $R_{1} L R_{3}, S_{1} L_{4}$ are the integration constants which are obtained by substituting the Eqs. (13-14) in the boundary conditions. After substitution, a set of seven algebraic equations in terms of nondimensional system parameters and nondimensional eigenfrequency parameter $(\bar{\lambda})$ is obtained as:

$$
\left[\mathrm{U}_{\mathrm{ij}}\left(\bar{\lambda}^{\mathrm{m}}\right)\right]=\left[\begin{array}{rrrrr}
\mathrm{U}_{11}^{\mathrm{m}} & \mathrm{L} & \mathrm{L} & \mathrm{L} & \mathrm{U}_{17}^{\mathrm{m}} \\
\mathrm{M} & \mathrm{O} & & & \mathrm{M} \\
\mathrm{M} & & \mathrm{O} & & \mathrm{M} \\
\mathrm{M} & & & \mathrm{O} & \mathrm{M} \\
\mathrm{U}_{71}^{\mathrm{m}} & \mathrm{L} & \mathrm{L} & \mathrm{L} & \mathrm{U}_{77}^{\mathrm{m}}
\end{array}\right]\left\{\begin{array}{l}
\mathrm{R}_{1}^{\mathrm{m}} \\
\mathrm{R}_{2}^{\mathrm{m}} \\
\mathrm{R}_{3}^{\mathrm{m}} \\
\mathrm{S}_{1}^{\mathrm{m}} \\
\mathrm{S}_{2}^{\mathrm{m}} \\
\mathrm{S}_{3}^{\mathrm{m}} \\
\mathrm{S}_{4}^{\mathrm{m}}
\end{array}\right\}=\left[\begin{array}{l}
0 \\
0 \\
0 \\
0 \\
0 \\
0 \\
0
\end{array}\right]
$$

The transcendental eigenfrequency equation in terms of known system parameters is obtained for the existence of non-trivial solution for the Eq. (15), i.e. $|\mathrm{U}(\bar{\lambda})|=0$; here $\mathrm{U}(\bar{\lambda})$ denotes the coefficient matrix of Eq. (16). Now, in order to obtain the eigenfunctions of the links obtained in Eqs. (13-14), the integration constants $\left(\mathrm{R}_{2}-\mathrm{R}_{3}, \mathrm{~S}_{1}-\mathrm{S}_{4}\right)^{\mathrm{m}}$ are expressed in terms of unit magnitude of $\mathrm{R}_{1}^{\mathrm{m}}$ and their respective expressions along with the elements of matrix $\left[\mathrm{U}_{\mathrm{ij}}\right]$ and nondimensional parameters are given in appendix.

\section{3: Nonlinear Analysis: problem formulation}

The nonlinear behaviors and stability characteristics of the two-link flexible manipulator with harmonically driven prismatic and revolute motion of frequency $\bar{\omega}_{1,2}$ have been analyzed. The nonlinear coupled terms and the cubic nonlinearity arising due to geometric stretching have been retained in the governing equations of motions of the links expressed in Eqs. (3-5) and viscous damping $\left(\mathrm{c}_{1,2}\right)$ is included in both the links.

For link 1:

$\rho_{1} \mathrm{~A}_{1}+\mathrm{E}_{1} \mathrm{I}_{1} \mathrm{~s}_{1}^{\prime \prime \prime}-(3 / 2) \mathrm{E}_{1} \mathrm{~A}_{1} \mathrm{~s}_{1}^{\prime 2} \mathrm{~s}_{1}^{\prime \prime}+\mathrm{c}_{1} \&=0$.

For link 2:

$$
\begin{aligned}
& \rho_{2} \mathrm{~A}_{2}\left(\eta+\eta+\mathrm{E}_{2} \mathrm{I}_{2} \mathrm{~s}_{2}^{\prime \prime \prime}-(3 / 2) \mathrm{E}_{2} \mathrm{~A}_{2} \mathrm{~s}_{2}^{\prime 2} \mathrm{~s}_{2}^{\prime \prime}+\mathrm{c}_{2} \&+\right.
\end{aligned}
$$

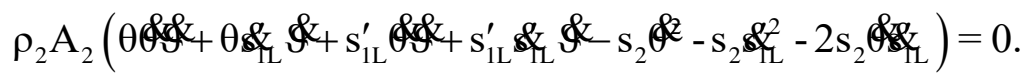


Nondimensionalization of Eqs. (16-17) has been established using the dimensionless terms: $\overline{\mathrm{s}}_{1}=\mathrm{s}_{1} / \mathrm{L}_{1}, \quad \overline{\mathrm{s}}_{2}=\mathrm{s}_{2} / \mathrm{L}_{2}, \quad \bar{\eta}=\eta / \mathrm{L}_{1}, \quad \overline{\mathrm{c}}_{2}=\mathrm{c}_{2} \mathrm{~L}_{1}^{2} / \alpha_{\mathrm{M}} \sqrt{\rho_{1} \mathrm{~A}_{1} \mathrm{E}_{1} \mathrm{I}_{1}}, \quad \overline{\mathrm{c}}_{1}=\mathrm{c}_{1} \mathrm{~L}_{1}^{2} / \sqrt{\rho_{1} \mathrm{~A}_{1} \mathrm{E}_{1} \mathrm{I}_{1}} \quad$ and $\tau=\mathrm{t} \sqrt{\mathrm{E}_{1} \mathrm{I}_{1} / \rho_{1} \mathrm{~A}_{1} \mathrm{~L}_{1}^{4}}$, and expressed as.

For link 1:

$\mathrm{s}_{1}^{*}+\overrightarrow{\mathrm{s}}_{1}^{\prime \prime \prime}-\left(3 \mathrm{~A}_{1} \mathrm{~L}_{1}^{2} / 2 \mathrm{I}_{1}\right) \overrightarrow{\mathrm{s}}_{1}^{2} \overrightarrow{\mathrm{s}}_{1}^{\prime \prime}+\mathrm{c}_{1} \mathrm{~s}_{1}^{\&}=0$.

For link 2:

$$
\begin{aligned}
& \mathrm{s}_{2}^{\mathrm{K}}+\left(\bar{\eta} / \alpha_{\mathrm{L}}\right) \mathrm{sL}_{1 \mathrm{~L}}^{\mathrm{K}}+\left(\bar{\eta} / \alpha_{\mathrm{L}}\right)+\left(\chi / \alpha_{\mathrm{M}}\right) \overrightarrow{\mathrm{s}}_{2}^{\prime \prime \prime}-\left(\chi / \alpha_{\mathrm{M}}\right)\left(3 \mathrm{~A}_{2} \mathrm{~L}_{2}^{2} / 2 \mathrm{I}_{2}\right) \overrightarrow{\mathrm{s}}_{2}^{2} \overrightarrow{\mathrm{s}}_{2}^{\prime \prime}+\mathrm{c}_{2} \mathrm{~s}_{2}^{\mathrm{\&}}
\end{aligned}
$$

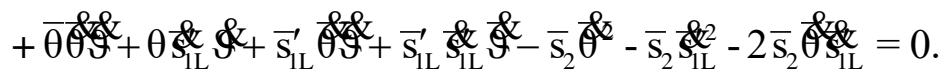

In further text, prime and over dot shall be read as the differentiation with respect to space parameter $\bar{\eta}$ and time $\tau$, respectively. Now, Galerkin's method is used to discretize spatiotemporal Eqs. (18-19) into temporal equations by using the expressions, $\overline{s_{1}}(\bar{\eta}, \tau)=h \varphi_{1}(\bar{\eta}) \mathrm{q}_{1}(\tau)$ and $\bar{s}_{2}(\bar{\eta}, \tau)=h \varphi_{2}(\bar{\eta}) \mathrm{q}_{2}(\tau)$. Here, $\mathrm{q}_{1}(\tau)$ and $\mathrm{q}_{2}(\tau)$ are the time modulation for the first and second link, respectively, $h$ is the scaling factor and $\varphi_{1}(\bar{\eta})$, and $\varphi_{2}(\bar{\eta})$ are the eigenfunction of the first and second links for first mode of vibrations, respectively given in Eqs. (13-14) and expressed here as:

$$
\begin{aligned}
& \varphi_{1}(\bar{\eta})=\mathrm{R}_{1} \mathrm{c}(\bar{\lambda} \bar{\eta})+\mathrm{R}_{2}\{\mathrm{~s}(\bar{\lambda} \bar{\eta})-\mathrm{sh}(\bar{\lambda} \bar{\eta})\}+\mathrm{R}_{3} \operatorname{ch}(\bar{\lambda} \bar{\eta}) \\
& \varphi_{2}(\bar{\eta})=\mathrm{S}_{1} \mathrm{c}(\kappa \bar{\lambda} \bar{\eta})+\mathrm{S}_{2} \mathrm{~s}(\kappa \bar{\lambda} \bar{\eta})+\mathrm{S}_{3} \operatorname{ch}(\kappa \bar{\lambda} \bar{\eta})+\mathrm{S}_{4} \operatorname{sh}(\kappa \bar{\lambda} \bar{\eta})
\end{aligned}
$$

The coefficient matrix of Eq. (15) is numerically solved for the given nondimensional system parameters to determine the nondimensional eigen frequency parameter, $\bar{\gamma}$ and the expressions for $R_{1}-G_{3}$, and $S_{1}-S_{4}$ are given in the appendix. Now, first using the orthogonal property of the mode shapes and then using a small book keeping parameter $(\varepsilon)$ for ordering the Eqs. (20-21), the resultant non-dimensional nonlinear second order ordinary differential equations of motion of the links are obtained as:

$$
\begin{aligned}
& \oiint(\tau)+\bar{\Omega}_{1}^{2} \mathrm{q}_{1}(\tau)+2 \varepsilon \mu_{1} \&-\varepsilon \beta_{1} \bar{\omega}_{1}^{2} \cos \left(\bar{\omega}_{1} \tau\right)-\varepsilon^{2} \beta_{2} \mathrm{q}_{1}^{3}(\tau)=0 \text {. } \\
& (\tau)+\bar{\Omega}_{2}^{2} \mathrm{q}_{2}(\tau)+2 \varepsilon \mu_{2} \alpha_{2}(\tau)-\varepsilon\left\{\beta_{3} \omega_{1}^{2} \cos \left(\bar{\omega}_{1} \tau\right)+\beta_{11} \bar{\omega}_{2}^{2} \cos \left(\bar{\omega}_{2} \tau\right)\right\}+ \\
& \varepsilon^{2}\left\{\begin{array}{l}
\beta_{4} \bar{\omega}_{1} \bar{\omega}_{2} \sin \left(\bar{\omega}_{1} \tau\right) \sin \left(\bar{\omega}_{2} \tau\right) \cos \left(\bar{\omega}_{2} \tau\right)-\beta_{5} \bar{\omega}_{1} \phi(\tau) \sin \left(\bar{\omega}_{1} \tau\right) \cos \left(\bar{\omega}_{2} \tau\right)+ \\
\beta_{12}(\tau)+\beta_{6} \bar{\omega}_{1} \bar{\omega}_{2} \mathrm{q}_{1}(\tau) \sin \left(\bar{\omega}_{1} \tau\right) \sin \left(\bar{\omega}_{2} \tau\right)-\beta_{7} \bar{\omega}_{1} \mathrm{q}_{1}(\tau) \notin(\tau) \sin \left(\bar{\omega}_{1} \tau\right)- \\
\beta_{8} \bar{\omega}_{2}^{2} \mathrm{q}_{2}(\tau) \sin ^{2}\left(\bar{\omega}_{1} \tau\right)-\beta_{9} \mathrm{q}_{2}(\tau) \oiint_{\tau}(\tau)+\beta_{10} \bar{\omega}_{2} \mathrm{q}_{2}(\tau) \notin(\tau) \sin \left(\bar{\omega}_{2} \tau\right)-\beta_{13} \mathrm{q}_{2}^{3}(\tau)
\end{array}\right\}=0 .
\end{aligned}
$$

The approximate solutions of Eqs. (22-23) are determined by using second order method and $\mathrm{q}_{1,2}$ are expressed in terms of fast $\left(\mathrm{T}_{0}=\tau\right)$ and slow time $\left(\mathrm{T}_{1}=\varepsilon \tau, \mathrm{T}_{2}=\varepsilon^{2} \tau\right)$ scales as: $\mathrm{q}_{1}=\mathrm{q}_{10}\left(\mathrm{~T}_{0}, \mathrm{~T}_{1}, \mathrm{~T}_{2}\right)+\varepsilon \mathrm{q}_{11}\left(\mathrm{~T}_{0}, \mathrm{~T}_{1}, \mathrm{~T}_{2}\right)+\varepsilon^{2} \mathrm{q}_{12}\left(\mathrm{~T}_{0}, \mathrm{~T}_{1}, \mathrm{~T}_{2}\right)$

$\mathrm{q}_{2}=\mathrm{q}_{20}\left(\mathrm{~T}_{0}, \mathrm{~T}_{1}, \mathrm{~T}_{2}\right)+\varepsilon \mathrm{q}_{21}\left(\mathrm{~T}_{0}, \mathrm{~T}_{1}, \mathrm{~T}_{2}\right)+\varepsilon^{2} \mathrm{q}_{22}\left(\mathrm{~T}_{0}, \mathrm{~T}_{1}, \mathrm{~T}_{2}\right)$

Using chain rule, time derivatives in terms of $\mathrm{T}_{0}, \mathrm{~T}_{1}$ and $\mathrm{T}_{2}$ become: 


$$
\begin{aligned}
& \mathrm{d} / \mathrm{dt}=\partial / \partial \mathrm{T}_{0}+\varepsilon \partial / \partial \mathrm{T}_{1}+\varepsilon^{2} \partial / \partial \mathrm{T}_{2} \\
& \mathrm{~d}^{2} / \mathrm{dt}^{2}=\partial^{2} / \partial \mathrm{T}_{0}^{2}+2 \varepsilon\left(\partial^{2} / \partial \mathrm{T}_{0} \partial \mathrm{T}_{1}\right)+\varepsilon^{2}\left(2\left(\partial^{2} / \partial \mathrm{T}_{0} \partial \mathrm{T}_{2}\right)+\partial^{2} / \partial \mathrm{T}_{1}^{2}\right)
\end{aligned}
$$

The equations of motion in different time scales for first link are obtained by substituting Eqs. (24-26) into Eq. (22) and after equating the coefficients of the same powers of $\varepsilon$ as:

$$
\begin{aligned}
& \mathrm{O}\left(\varepsilon^{1}\right): \partial^{2} \mathrm{q}_{10} / \partial \mathrm{T}_{0}^{2}+\bar{\Omega}_{1}^{2} \mathrm{q}_{10}=0 \\
& \mathrm{O}\left(\varepsilon^{2}\right): \partial^{2} \mathrm{q}_{11} / \partial \mathrm{T}_{1}^{2}+\bar{\Omega}_{1}^{2} \mathrm{q}_{11}+2 \mu_{1}\left(\partial \mathrm{q}_{10} / \partial \mathrm{T}_{0}\right)+2\left(\partial^{2} \mathrm{q}_{10} / \partial \mathrm{T}_{0} \partial \mathrm{T}_{1}\right)-\beta_{1} \bar{\omega}_{1}^{2} \cos \left(\bar{\omega}_{1} \mathrm{~T}_{0}\right)=0 \text {. }
\end{aligned}
$$

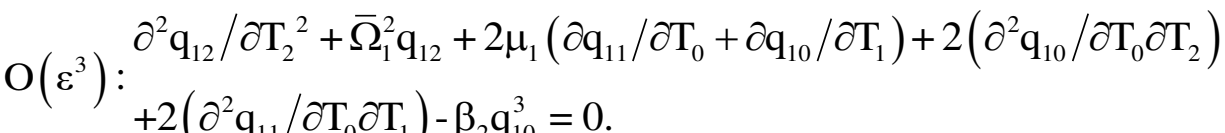

The equations for second link are obtained in a similar manner as:

$$
\begin{aligned}
\mathrm{O}\left(\varepsilon^{1}\right): & \partial^{2} \mathrm{q}_{20} / \partial \mathrm{T}_{0}^{2}+\bar{\Omega}_{2}^{2} \mathrm{q}_{20}=0 \\
\mathrm{O}\left(\varepsilon^{2}\right): & \partial^{2} \mathrm{q}_{21} / \partial \mathrm{T}_{1}^{2}+\bar{\Omega}_{2}^{2} \mathrm{q}_{21}+2 \mu_{2}\left(\partial \mathrm{q}_{20} / \partial \mathrm{T}_{0}\right)+2\left(\partial^{2} \mathrm{q}_{20} / \partial \mathrm{T}_{0} \partial \mathrm{T}_{1}\right)- \\
& \beta_{3} \bar{\omega}_{1}^{2} \cos \left(\bar{\omega}_{1} \mathrm{~T}_{0}\right)-\beta_{11} \bar{\omega}_{1}^{2} \cos \left(\bar{\omega}_{2} \mathrm{~T}_{0}\right)=0 . \\
& \partial^{2} \mathrm{q}_{22} / \partial \mathrm{T}_{2}^{2}+\bar{\Omega}_{2}^{2} \mathrm{q}_{22}+2 \mu_{2}\left(\partial \mathrm{q}_{21} / \partial \mathrm{T}_{0}+\partial \mathrm{q}_{20} / \partial \mathrm{T}_{1}\right)+2\left(\partial^{2} \mathrm{q}_{20} / \partial \mathrm{T}_{0} \partial \mathrm{T}_{2}\right)+ \\
& 2\left(\partial^{2} \mathrm{q}_{21} / \partial \mathrm{T}_{0} \partial \mathrm{T}_{1}\right)+\partial^{2} \mathrm{q}_{20} / \partial \mathrm{T}_{1}^{2}+\beta_{12} \partial^{2} \mathrm{q}_{10} / \partial \mathrm{T}_{0}^{2}-\beta_{3} \omega_{1}^{2} \cos \left(\bar{\omega}_{1} \mathrm{~T}_{0}\right)-\beta_{11} \bar{\omega}_{2}^{2} \cos \left(\bar{\omega}_{2} \mathrm{~T}_{0}\right) \\
\mathrm{O}\left(\varepsilon^{3}\right): & +\beta_{4} \bar{\omega}_{1} \bar{\omega}_{2} \sin \left(\bar{\omega}_{1} \mathrm{~T}_{0}\right) \sin \left(\bar{\omega}_{2} \mathrm{~T}_{0}\right) \cos \left(\bar{\omega}_{2} \mathrm{~T}_{0}\right)-\beta_{5} \bar{\omega}_{1}\left(\partial \mathrm{q}_{10} / \partial \mathrm{T}_{0}\right) \sin \left(\bar{\omega}_{1} \mathrm{~T}_{0}\right) \cos \left(\bar{\omega}_{2} \mathrm{~T}_{0}\right)+ \\
& \beta_{6} \bar{\omega}_{1} \bar{\omega}_{2} \mathrm{q}_{10} \sin \left(\bar{\omega}_{1} \mathrm{~T}_{0}\right) \sin \left(\bar{\omega}_{2} \mathrm{~T}_{0}\right)-\beta_{7} \bar{\omega}_{1} \mathrm{q}_{10}\left(\partial \mathrm{q}_{10} / \partial \mathrm{T}_{0}\right) \sin \left(\bar{\omega}_{1} \mathrm{~T}_{0}\right)-\beta_{8} \bar{\omega}_{2}^{2} \mathrm{q}_{20} \sin ^{2}\left(\bar{\omega}_{1} \mathrm{~T}_{0}\right) \\
& -\beta_{9} \mathrm{q}_{20}\left(\partial \mathrm{q}_{10} / \partial \mathrm{T}_{0}\right)^{2}+\beta_{10} \bar{\omega}_{2} \mathrm{q}_{20}\left(\partial \mathrm{q}_{10} / \partial \mathrm{T}_{0}\right) \sin \left(\bar{\omega}_{2} \mathrm{~T}_{0}\right)-\beta_{13} \mathrm{q}_{20}^{3}=0 .
\end{aligned}
$$

The general solution of differential equations Eqs. (27 \& 30) can be expressed as:

$$
\begin{aligned}
& \mathrm{q}_{10}=\overline{\mathrm{Q}}_{1}\left(\mathrm{~T}_{1}, \mathrm{~T}_{2}\right) \exp \left(\mathrm{i} \bar{\Omega}_{1} \mathrm{~T}_{0}\right)+\overline{\mathrm{Q}}_{1}\left(\mathrm{~T}_{1}, \mathrm{~T}_{2}\right) \exp \left(-\mathrm{i} \bar{\Omega}_{1} \mathrm{~T}_{0}\right) \\
& \mathrm{q}_{20}=\overline{\mathrm{Q}}_{2}\left(\mathrm{~T}_{1}, \mathrm{~T}_{2}\right) \exp \left(\mathrm{i} \bar{\Omega}_{2} \mathrm{~T}_{0}\right)+\overline{\mathrm{Q}}_{2}\left(\mathrm{~T}_{1}, \mathrm{~T}_{2}\right)\left(-\mathrm{i} \bar{\Omega}_{2} \mathrm{~T}_{0}\right)
\end{aligned}
$$

Now, it can be observed that any solution of Eq. $(28,31)$ will contain secular or small divisor terms if the frequency of prismatic joint motion $\left(\bar{\omega}_{1}\right)$ and revolute joint $\left(\bar{\omega}_{2}\right)$ motion become equal or nearly equal to link's normalized frequencies $\left(\bar{\Omega}_{1,2}\right)$. These small divisor terms lead to unbounded solutions. In order to have bounded solutions of the respective equations these secular or small divisor terms should be removed. Also, it has been numerically found that the normalized link frequencies $\left(\bar{\Omega}_{1,2}\right)$ are nearly equal for same link parameters which results in the existence of internal resonance between the links of the manipulator.

2.3.1. Primary resonance case in first and second link: $\left(\bar{\omega}_{1} \approx \bar{\Omega}_{1}\right.$ and $\left.\bar{\omega}_{2} \approx \bar{\Omega}_{2}\right)$

In case of first link, the simple resonance condition exits when the frequency of prismatic motion $\left(\bar{\omega}_{1}\right)$ becomes equal or nearly equal to the normalized frequency $\left(\bar{\Omega}_{1}\right)$ and in second link when the frequency of revolute joint motion $\left(\bar{\omega}_{2}\right)$ becomes equal or nearly equal to the normalized 
frequency $\left(\bar{\Omega}_{2}\right)$. Now, for simple resonance case, the nearness of $\bar{\omega}_{1}$ to $\bar{\Omega}_{1}$ and $\bar{\omega}_{2}$ to $\bar{\Omega}_{2}$ are respectively expressed as, $\bar{\Omega}_{1}+\varepsilon \sigma_{1}$, and $\bar{\Omega}_{2}+\varepsilon \sigma_{2}$, here $\sigma_{1}$ and $\sigma_{2}$ are known as detuning parameters. Following a similar procedure as adopted in Kumar and Pratiher [11], we obtain the governing equations for the vibration for both the links in terms of original time variable $\tau$ as:

$$
\begin{aligned}
& 2 \mathrm{i} \bar{\Omega}_{1}\left(\partial \mathrm{Q}_{1} / \partial \tau\right)+2 \mathrm{i} \bar{\Omega}_{1} \varepsilon \mu_{1} \mathrm{Q}_{1}+\left\{\left(\beta_{1} \varepsilon^{2} \bar{\omega}_{1}^{2} \sigma_{1} / 4 \bar{\Omega}_{1}\right)-\left(\beta_{1} \varepsilon \bar{\omega}_{1}^{2} / 2\right)\right\} \exp \left(\mathrm{i} \sigma_{1} \mathrm{~T}_{1}\right)- \\
& \left(\mathrm{i} \beta_{1} \varepsilon^{2} \bar{\omega}_{1}^{2} \mu_{1} / 4 \bar{\Omega}_{1}\right) \exp \left(\mathrm{i} \sigma_{1} \mathrm{~T}_{1}\right)-3 \beta_{2} \varepsilon^{2} \mathrm{Q}_{1}^{2} \overline{\mathrm{Q}}_{1}-\varepsilon^{2} \mu_{1}^{2} \mathrm{Q}_{1}=0 . \\
& 2 \mathrm{i} \bar{\Omega}_{2}\left(\partial \mathrm{Q}_{2} / \partial \tau\right)+2 \mathrm{i} \bar{\Omega}_{2} \varepsilon \mu_{2} \mathrm{Q}_{2}+\left\{\left(\beta_{11} \varepsilon^{2} \bar{\omega}_{2}^{2} \sigma_{2} / 4 \bar{\Omega}_{2}\right)-\left(\beta_{11} \varepsilon \bar{\omega}_{2}^{2} / 2\right)\right\} \exp \left(\mathrm{i} \sigma_{2} \mathrm{~T}_{1}\right)-2 \beta_{9} \varepsilon^{2} \bar{\Omega}_{1}^{2} \mathrm{Q}_{1} \overline{\mathrm{Q}}_{1} \mathrm{Q}_{2}- \\
& \left(\mathrm{i} \beta_{11} \varepsilon^{2} \bar{\omega}_{2}^{2} \mu_{2} / 4 \bar{\Omega}_{2}\right) \exp \left(\mathrm{i}_{2} \mathrm{~T}_{1}\right)-\left(\beta_{8} \varepsilon^{2} \bar{\omega}_{2}^{2} / 2\right)\left(\mathrm{Q}_{2}-\overline{\mathrm{Q}}_{2} \exp \left(2 \mathrm{i} \sigma_{2} \mathrm{~T}_{1}\right) / 2\right)-3 \beta_{13} \varepsilon^{2} \mathrm{Q}_{2}^{2} \overline{\mathrm{Q}}_{2}-\varepsilon^{2} \mu_{2}{ }^{2} \mathrm{Q}_{2}=0 .
\end{aligned}
$$

Substituting the polar form of $\mathrm{Q}_{1}(\tau)$ and $\mathrm{Q}_{2}(\tau)$ as $\mathrm{Q}_{1}(\tau)=(1 / 2) \mathrm{r}_{1}(\tau) \mathrm{e}^{\mathrm{i}_{1}(\tau)}$ and $\mathrm{Q}_{2}(\tau)=(1 / 2) \mathrm{r}_{2}(\tau) \mathrm{e}^{\mathrm{i} \phi_{2}(\tau)}$ and separating real and imaginary parts, the following differential equations are obtained by letting $\delta_{1}=\sigma_{1} \mathrm{~T}_{1}-\varphi_{1}$ and $\delta_{2}=\sigma_{2} \mathrm{~T}_{1}-\phi_{2}$ :

$$
\begin{aligned}
& \bar{\Omega}_{1}\left(\partial \mathrm{r}_{1} / \partial \tau\right)+\bar{\Omega}_{1} \varepsilon \mu_{1} \mathrm{r}_{1}+\left\{\beta_{1} \varepsilon^{2} \bar{\omega}_{1}^{2} \sigma_{1} / 4 \bar{\Omega}_{1}-\beta_{1} \varepsilon \bar{\omega}_{1}^{2} / 2\right\} \mathrm{s}\left(\delta_{1}\right)-\left(\beta_{1} \varepsilon^{2} \bar{\omega}_{1}^{2} \mu_{1} / 4 \bar{\Omega}_{1}\right) \mathrm{c}\left(\delta_{1}\right)=0 . \\
& \mathrm{r}_{1} \bar{\Omega}_{1}\left(\partial \delta_{1} / \partial \tau\right)-\varepsilon \sigma_{1} \mathrm{r}_{1} \bar{\Omega}_{1}+\left\{\beta_{1} \varepsilon^{2} \bar{\omega}_{1}^{2} \sigma_{1} / 4 \bar{\Omega}_{1}-\beta_{1} \varepsilon \bar{\omega}_{1}^{2} / 2\right\} \mathrm{c}\left(\delta_{1}\right)+\left(\beta_{1} \varepsilon^{2} \bar{\omega}_{1}^{2} \mu_{1} / 4 \bar{\Omega}_{1}\right) \mathrm{s}\left(\delta_{1}\right)- \\
& \varepsilon \mu_{1}^{2} \mathrm{r}_{1} / 2-(3 / 8) \beta_{2} \varepsilon^{2} \mathrm{r}_{1}^{3}=0 . \\
& \bar{\Omega}_{2}\left(\partial \mathrm{r}_{2} / \partial \tau\right)+\bar{\Omega}_{2} \varepsilon \mu_{2} \mathrm{r}_{2}+\left\{\beta_{11} \varepsilon^{2} \bar{\omega}_{2}^{2} \sigma_{2} / 4 \bar{\Omega}_{2}-\beta_{11} \varepsilon \bar{\omega}_{2}^{2} / 2\right\} \mathrm{s}\left(\delta_{2}\right)- \\
& \left(\beta_{11} \varepsilon^{2} \bar{\omega}_{2}^{2} \mu_{2} / 4 \bar{\Omega}_{2}\right) \mathrm{c}\left(\delta_{2}\right)+\left(\beta_{8} \varepsilon^{2} \bar{\omega}_{2}^{2} / 8\right) \mathrm{r}_{2} \mathrm{~s}\left(2 \delta_{2}\right)=0 . \\
& \mathrm{r}_{2} \bar{\Omega}_{2}\left(\partial \delta_{1} / \partial \tau\right)-\varepsilon \sigma_{2} \mathrm{r}_{2} \bar{\Omega}_{2}+\left\{\beta_{11} \varepsilon^{2} \bar{\omega}_{2}^{2} \sigma_{2} / 4 \bar{\Omega}_{2}-\beta_{11} \varepsilon \bar{\omega}_{2}^{2} / 2\right\} \mathrm{c}\left(\delta_{2}\right)+\left(\beta_{11} \varepsilon^{2} \bar{\omega}_{2}^{2} \mu_{2} / 4 \bar{\Omega}_{2}\right) \mathrm{s}\left(\delta_{2}\right) \\
& +\left(\beta_{8} \varepsilon^{2} \bar{\omega}_{2}^{2} / 8\right) \mathrm{r}_{2} \mathrm{c}\left(2 \delta_{2}\right)-\left\{\varepsilon \mu_{2}^{2} / 2+\varepsilon^{2} \beta_{8} \bar{\omega}_{2}^{2} / 2+\beta_{9} \varepsilon^{2} \bar{\Omega}_{1}^{2} \mathrm{r}_{1}^{2} / 8\right\} \mathrm{r}_{2}-(3 / 8) \beta_{13} \varepsilon^{2} \mathrm{r}_{2}^{3}=0 .
\end{aligned}
$$

The solutions of first and second link up to the second order approximations in terms of original time variable $\tau$, can be expressed as:

$$
\begin{aligned}
& \mathrm{q}_{1}=(1 / 2) \mathrm{r}_{1} \cos \left(\bar{\omega}_{1} \tau-\delta_{1}\right)+\mathrm{O}\left(\varepsilon^{2}\right), \\
& \mathrm{q}_{2}=(1 / 2) \mathrm{r}_{2} \cos \left(\bar{\omega}_{2} \tau-\delta_{2}\right)+\varepsilon\left\{\beta_{3} \bar{\omega}_{1}^{2} / 2\left(\bar{\Omega}_{2}^{2}-\bar{\omega}_{1}^{2}\right)\right\} \cos \left(\bar{\omega}_{1} \tau\right)+\mathrm{O}\left(\varepsilon^{2}\right) .
\end{aligned}
$$

The steady state response of Eqs. (37-40) can be obtained by equating $\left(\partial \mathrm{r}_{\mathrm{j}} / \partial \tau\right)$ and $\left(\partial \delta_{\mathrm{j}} / \partial \tau\right)$ $(j=1,2)$ to be zero which results in a set of two equations for both the links. Further, the respective frequency response equations of the links in terms of $r_{j}$ and $\sigma_{j}$ can be obtained by eliminating $\delta_{\mathrm{j}}$ from the resultant equations obtained earlier. For the first link, in order to determine the stability of the steady state solutions, a small perturbation is introduced in Eqs. (37-38), and $\mathrm{r}_{1}$, and $\delta_{1}$ are replaced with $\mathrm{r}_{1}=\mathrm{r}_{10}+\mathrm{r}_{11}$, and $\delta_{1}=\delta_{10}+\delta_{11}$. After linearizing, the Eqs. (37-38) are arranged in matrix form as: 


$$
\left\{\begin{array}{c}
\& \\
\& \\
\&
\end{array}\right\}=\left[\begin{array}{cl}
-\varepsilon \mu_{1} & -\left\{\beta_{1} \varepsilon^{2} \bar{\omega}_{1}^{2} \sigma_{1} / 4 \bar{\Omega}_{1}^{2}-\beta_{1} \varepsilon \bar{\omega}_{1}^{2} / 2 \bar{\Omega}_{1}\right\} \mathrm{c}\left(\delta_{10}\right)- \\
& \left(\beta_{1} \varepsilon^{2} \bar{\omega}_{1}^{2} \mu_{1} / 4 \bar{\Omega}_{1}^{2}\right) \mathrm{s}\left(\delta_{10}\right) \\
& \left\{\beta_{1} \varepsilon^{2} \bar{\omega}_{1}^{2} \sigma_{1} / 4 \mathrm{r}_{10} \bar{\Omega}_{1}^{2}-\beta_{1} \varepsilon \bar{\omega}_{1}^{2} / 2 \mathrm{r}_{10} \bar{\Omega}_{1}\right\} \mathrm{s}\left(\delta_{10}\right)- \\
\varepsilon \sigma_{1} / \mathrm{r}_{10}+9 \varepsilon^{2} \beta_{2} \mathrm{r}_{10} / 8+\varepsilon \mu_{1}^{2} / 2 \mathrm{a}_{10} \lambda_{1} & \left(\beta_{1} \varepsilon^{2} \bar{\omega}_{1}^{2} \mu_{1} / 4 \mathrm{r}_{10} \bar{\Omega}_{1}^{2}\right) \mathrm{c}\left(\delta_{10}\right)
\end{array}\right]\left\{\begin{array}{c}
\mathrm{r}_{11} \\
\delta_{11}
\end{array}\right\}
$$

The equilibrium points $r_{10}$, and $\delta_{10}$ satisfy the steady state equations for first link and the stability of the steady state solutions depends on the nature of the eigenvalues of the coefficient matrix of Eq. (42). The steady state solutions will be stable if and only if the real parts of the eigenvalues are negative. The steady state solutions stability for the second link can also be determined in a similar manner.

2.3.2. Internal resonance in second link: $\left(\bar{\omega}_{1} \approx \bar{\Omega}_{1}\right.$, and $\left.\bar{\Omega}_{1} \approx \bar{\Omega}_{2}\right)$

Numerically, it was found that the normalized frequency of the first link $\left(\bar{\Omega}_{1}\right)$ is nearly equal to the normalized frequency of the second $\operatorname{link}\left(\bar{\Omega}_{2}\right)$ leading to the existence of internal resonance. Here, the primary resonance occurs in first link $\left(\bar{\omega}_{1} \approx \bar{\Omega}_{1}\right)$ and the second link vibrates due to the internal resonance between the links $\left(\bar{\Omega}_{1} \approx \bar{\Omega}_{2}\right)$. The nearness of the $\bar{\Omega}_{1}$ to $\bar{\Omega}_{2}$ is expressed as, $\bar{\Omega}_{2}+\varepsilon \sigma_{3}$ and $\sigma_{3}$ is detuning parameters for internal resonance. The governing equation of motion for the first link remains same as Eq. (22) and hence the equation for the vibrating amplitude and phase are given by Eq. (37-38). However, for second link the reduced equations for vibration amplitude and phase after following the procedure explained earlier are expressed as:

$$
\begin{aligned}
& \bar{\Omega}_{2}\left(\partial \mathrm{r}_{3} / \partial \tau\right)+\bar{\Omega}_{2} \varepsilon \mu_{2} \mathrm{r}_{3}+\left\{\beta_{3} \varepsilon^{2} \bar{\omega}_{1}^{2}\left(\sigma_{1}+\sigma_{3}\right) / 4 \bar{\Omega}_{2}-\beta_{3} \varepsilon \bar{\omega}_{1}^{2} / 2\right\} \mathrm{s}\left(\delta_{3}+\delta_{1}\right)-\left(\beta_{12} \varepsilon^{2} \bar{\Omega}_{1}^{2} \mathrm{r}_{1} / 2\right) \mathrm{s}\left(\delta_{3}\right)- \\
& \left(\beta_{3} \varepsilon^{2} \bar{\omega}_{1}^{2} \mu_{2} / 4 \bar{\Omega}_{2}\right) \mathrm{c}\left(\delta_{3}+\delta_{1}\right)+\left(\beta_{9} \varepsilon^{2} \bar{\Omega}_{1}^{2} \mathrm{r}_{1}^{2} / 8\right) \mathrm{r}_{3} \mathrm{~s}\left(2 \delta_{3}\right)+\left(\beta_{7} \varepsilon^{2} \bar{\Omega}_{1} \bar{\omega}_{1} \mathrm{r}_{1}^{2} / 8\right) \mathrm{s}\left(\delta_{3}-\delta_{1}\right)=0 . \\
& \mathrm{r}_{3} \bar{\Omega}_{2}\left(\partial \delta_{1} / \partial \tau+\partial \delta_{3} / \partial \tau\right)-\varepsilon\left(\sigma_{3}+\sigma_{1}\right) \mathrm{r}_{3} \bar{\Omega}_{2}+\left\{\beta_{3} \varepsilon^{2} \bar{\omega}_{1}^{2}\left(\sigma_{1}+\sigma_{3}\right) / 4 \bar{\Omega}_{2}-\beta_{3} \varepsilon \bar{\omega}_{1}^{2} / 2\right\} \mathrm{c}\left(\delta_{3}+\delta_{1}\right) \\
& +\left(\beta_{3} \varepsilon^{2} \bar{\omega}_{1}^{2} \mu_{2} / 4 \bar{\Omega}_{2}\right) \mathrm{s}\left(\delta_{3}+\delta_{1}\right)+\left(\beta_{9} \varepsilon^{2} \bar{\Omega}_{1}^{2} \mathrm{r}_{1}^{2} / 8\right) \mathrm{r}_{3} \mathrm{c}\left(2 \delta_{3}\right)+\left(\beta_{7} \varepsilon^{2} \bar{\Omega}_{1} \bar{\omega}_{1} \mathrm{r}_{1}^{2} / 8\right) \mathrm{c}\left(\delta_{3}-\delta_{1}\right)- \\
& \left(\beta_{12} \varepsilon^{2} \bar{\Omega}_{1}^{2} \mathrm{r}_{1} / 2\right) \mathrm{c}\left(\delta_{3}\right)-\left\{\varepsilon \mu_{2}^{2} / 2+\varepsilon^{2} \beta_{8} \bar{\omega}_{2}^{2} / 2+\beta_{9} \varepsilon^{2} \bar{\Omega}_{1}^{2} \mathrm{r}_{1}^{2} / 8\right\} \mathrm{r}_{3}-(3 / 8) \beta_{13} \varepsilon^{2} \mathrm{r}_{3}^{3}=0 .
\end{aligned}
$$

Here, $\delta_{1}=\sigma_{1} \mathrm{~T}_{1}-\varphi_{1}, \delta_{3}=\sigma_{3} \mathrm{~T}_{1}-\phi_{3}+\phi_{1}$ and second order solution for the second link motion is $\mathrm{q}_{3}=(1 / 2) \mathrm{r}_{3} \cos \left(\bar{\Omega}_{1} \tau-\delta_{3}\right)+\varepsilon\left\{\beta_{11} \bar{\omega}_{2}^{2} / 2\left(\bar{\Omega}_{2}^{2}-\bar{\omega}_{2}^{2}\right)\right\} \cos \left(\bar{\omega}_{2} \tau\right)+\mathrm{O}\left(\varepsilon^{2}\right)$. The frequency response equation for the internal resonance case can be obtained by eliminating phase $\left(\delta_{3}\right)$ for the steady state condition of Eqs. (43-44). The stability of the steady state solutions can be determined by introducing small perturbations in Eqs. (43-44) as explained in earlier section and the analyzing the eigenvalues of the coefficient matrix of resultant equations. 


\section{Numerical Results and Discussion}

\subsection{Modal analysis: Modal Parameters}

The eigenspectrums of a system are the essential parameters whose understanding is crucial for its safe operation and acceptable performance as well. The system vibrates at inadmissible amplitude leading to its failure or catastrophic injuries to the operator involved when the system is operated at a frequency equal or nearly equal to one of its natural frequencies. The variation of the eigenfrequencies with the system parameters provides a better understanding of the operational territory of the system in order to avoid such occurrences. The variation of system parameters has been accomplished by alteration of nondimensional parameters and thus apprehending its effect on the eigenspectrums of the two-link flexible manipulator with prismatic and revolute joints. The variation of payload mass parameter $\left(\alpha_{\mathrm{m} 2}\right)$ corresponds to the different payload masses being lifted by the terminal end of the link, the influence change in flexibility and masses of the links can be achieved respectively by varying the flexural rigidity $(\chi)$ of the system and the beam mass density ratio $\left(\alpha_{M}\right)$. A similar interpretation can be sought for the variation of other system parameters.
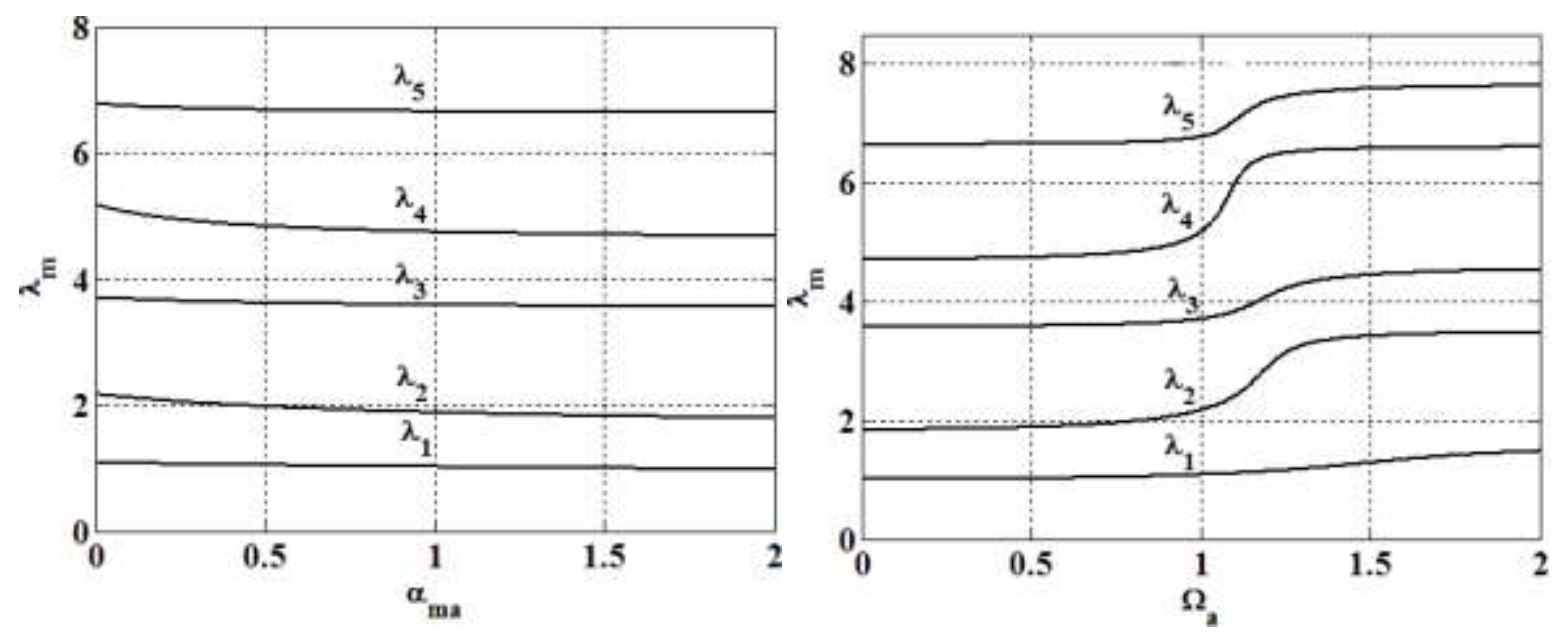

Fig. 2: Influence of actuator mass parameter $\left(\alpha_{\text {ma }}\right)$ and actuator frequency parameter $\left(\Omega_{a}\right)$ on eigenfrequencies of flexible two-link manipulator with prismatic and revolute joints for $\alpha_{\mathrm{m} 1,2}$ $=1.0, \chi=1.0, \alpha_{\mathrm{M}}=1.0, \alpha_{\mathrm{ma}}=1.0, \alpha_{\mathrm{J}}=1.0$, and $\Omega_{\mathrm{J}}=0.5$.

Effect of actuator mass parameter $\left(\alpha_{\mathrm{ma}}\right)$ and actuator frequency parameter $\left(\Omega_{\mathrm{a}}\right)$ on eigenfrequencies of flexible two-link manipulator with prismatic and revolute joints has been depicted in Fig. 2. Actuator frequency $\left(\Omega_{\mathrm{a}}\right)$ parameter represents the stiffness of the actuator if all other parameters are kept constant. It is evident that the system eigenfrequencies decrease as the inertia is added to the system $\left(; \omega_{\mathrm{n}} \propto \sqrt{\mathrm{k} / \mathrm{m}}\right)$ either by increasing the mass $\left(\alpha_{\mathrm{ma}}\right)$ or by decreasing the stiffness of actuator joint. It is also evident from the figure that the system experiences a jump into higher eigenfrequencies at the unit magnitude of the actuator frequency. 
As a result, manipulator may undergo higher mode of vibration. It has been noticed that adding stiffness to the system either by increasing the flexural rigidity $(\chi)$ or by increasing the stiffness of the rotary joint $\left(\Omega_{\mathrm{J}}\right)$ increases the overall eigenfrequency of the system. In addition, the unit magnitude of the joint frequency $\left(\Omega_{\mathrm{J}}\right)$ represents the condition when the natural frequency of the manipulator becomes equal to that of natural frequency of revolute joint comprising a lumpparameter model of torsional spring-inertia system depicting the flexible joint. At unit magnitude, joint dynamics gets decouple from the system and behaves as point mass. It has also been observed that the system eigenfrequency experience a sudden jump at unit magnitude of the joint frequency and hence, beyond this value the system starts oscillating at a higher mode of vibration. It is also observed that a lower eigenfrequency generates approximately at $\Omega_{2}=1.5$ and due to which the system again tends to vibrate at a lower eigenfrequency. These significant effects of the system dynamic characteristics on the fundamental eigenfrequency of the system may considerably influence the modal deflections and control parameters. In order to develop efficient control strategies and have a better understanding of actual dynamic responses of flexible robots, the development of accurate eigenspectrums is a perquisite requirement. The effect of system parameters on the dynamic responses is investigated through the eigenfunction which has been used to discretize the infinite dynamic model of the manipulator governed by the partial differential equations. Therefore, a brief investigation into the influence of system parameters on the eigenfunctions of the two-link flexible manipulator has been presented in Figs 3-6. The arrows in the figures indicate the decreases or increase in modal amplitude of the manipulator.
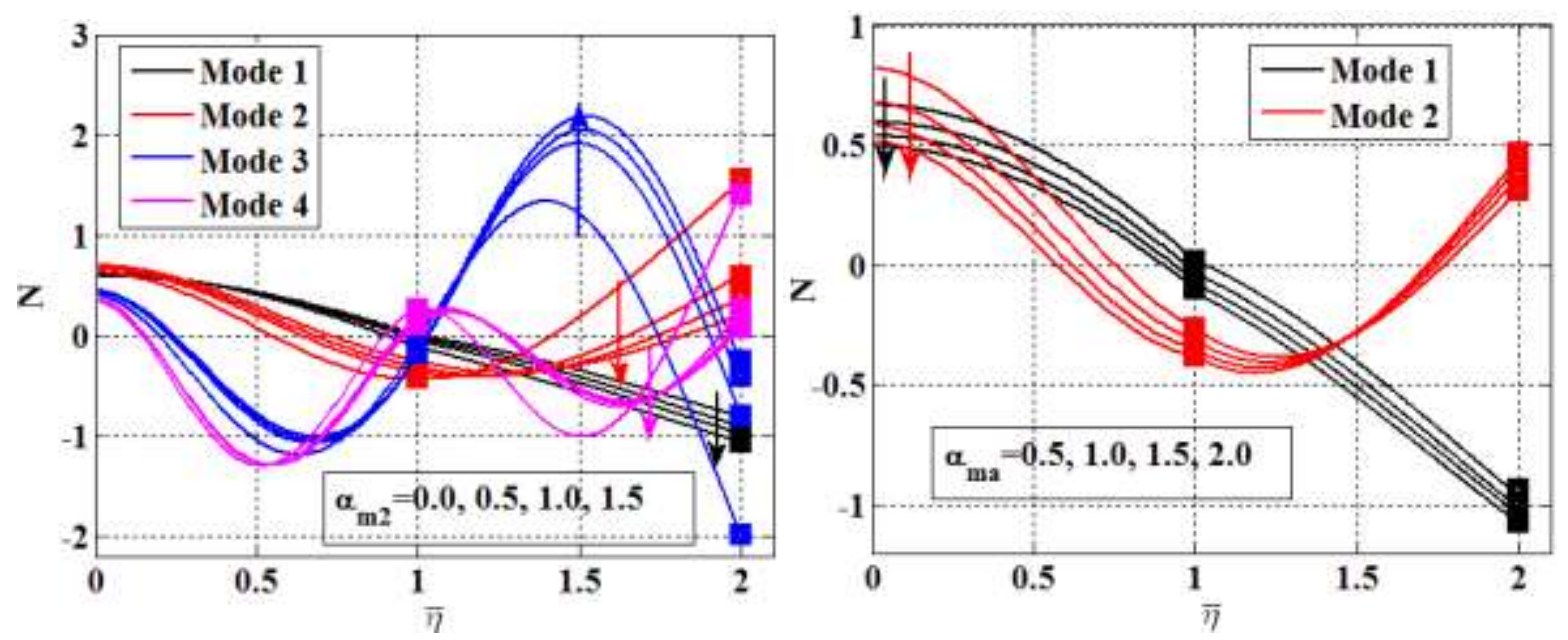

Fig. 3: Influence of payload and actuator mass parameter $\left(\alpha_{\mathrm{m} 2, \mathrm{a}}\right)$ on mode shapes of flexible two-link manipulator with prismatic and revolute joints with $\alpha_{\mathrm{m} 1}=1.0, \chi=1.0, \alpha_{\mathrm{M}}=1.0$, $\alpha_{\mathrm{J}}=1.0, \Omega_{\mathrm{a}, \mathrm{J}}=0.5$.

Fig. 3 depicts the variation of first four modes of vibration with the change in payload $\left(\alpha_{\mathrm{m} 2}\right)$ and actuator mass $\left(\alpha_{\mathrm{ma}}\right)$. The payload $\left(\alpha_{\mathrm{m} 2}\right)$ being lifted by the manipulator has a significant effect on the modal amplitude of the system. In comparison to no-load condition, modal amplitude for 
lower modes of vibration decreases as the mass of the payload $\left(\alpha_{\mathrm{m} 2}\right)$ at the terminal end of the manipulator is increased. However, the influence of payload $\left(\alpha_{\mathrm{m} 2}\right)$ is observed to be negligible on the higher modes of vibration while mode shapes tend to clutter along the length of the manipulator. The mass of the actuator $\left(\alpha_{\text {ma }}\right)$ majorly affects the modal amplitude of the roller supported end which is also evident from the figure. The modal amplitude at roller supported end decreases with the increase in actuator mass $\left(\alpha_{\text {ma }}\right)$ with an insignificant effect on modal parameter at terminal end.
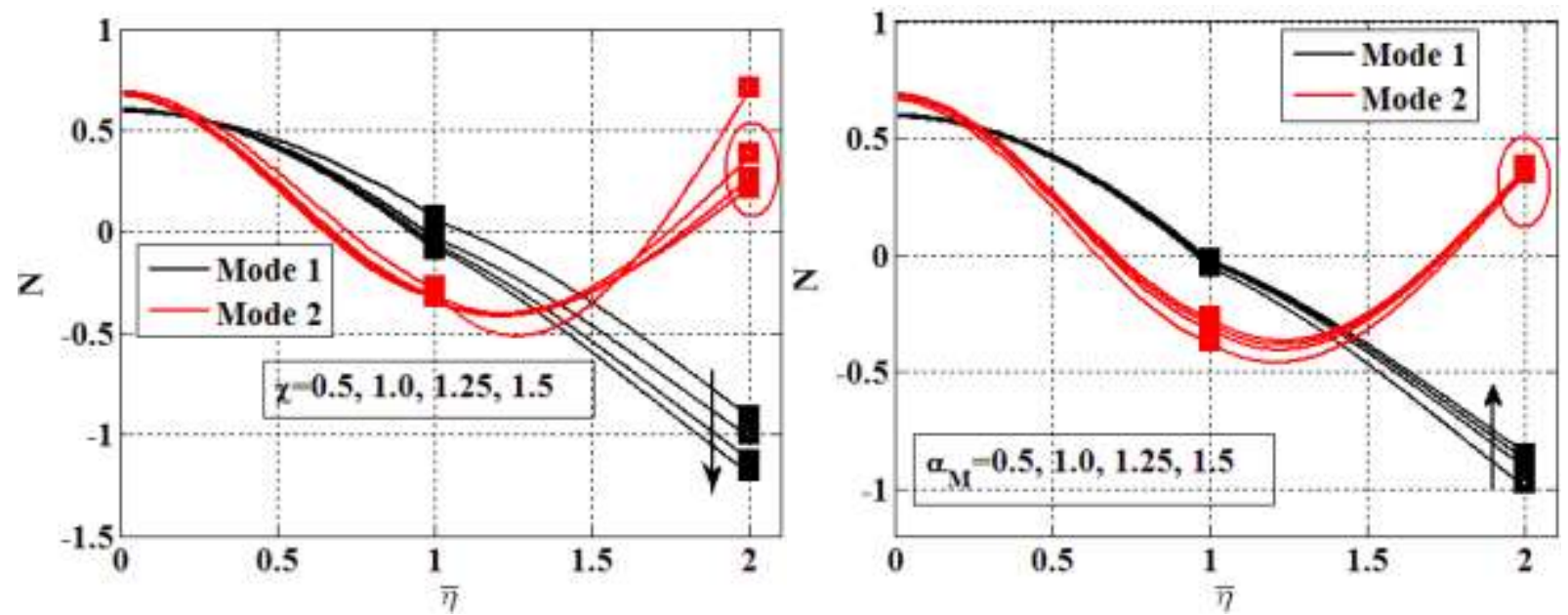

Fig. 4: Influence of flexural rigidity $(\chi)$ and beam mass density ratio $\left(\alpha_{M}\right)$ on mode shapes of flexible two-link manipulator with prismatic and revolute joints with $\alpha_{\mathrm{m} 1,2, \mathrm{a}}=1.0, \alpha_{\mathrm{J}}=1.0$, $\Omega_{\mathrm{a}, \mathrm{J}}=0.5$.

The flexural rigidity ratio $(\chi)$ representing the flexibility of the links can be varied by manipulating the cross-sectional dimensions of the links. The influence of flexural rigidity $(\chi)$ is significant on the lower modes of vibration as shown in Fig. 4. The amplitude of the manipulator decreases with the flexural rigidity ratio $(\chi)$ and the mode shapes tend spread out along the length of the manipulator. The beam mass density $\left(\alpha_{M}\right)$ is manipulated by appropriately varying the thickness of the links. From Fig. 4, it is also visible that the amplitude of vibration of manipulator decreases with increase in beam density $\left(\alpha_{M}\right)$. The influence of joint inertia parameter $\left(\alpha_{\mathrm{J}}\right)$ on the mode shapes of the flexible two-link manipulator is demonstrated in Fig. 5. The modal amplitude of manipulator increases with the increase in joint inertia $\left(\alpha_{\mathrm{J}}\right)$ and higher mode shapes tend to clutter along with its length. In Fig. 2, it has been observed that the second eigenfrequency $\left(\bar{\lambda}_{2}\right)$ witness a sudden jump at unit magnitude of the actuator frequency $\left(\Omega_{\mathrm{a}}=1\right)$ and hence the system vibrates at a higher mode of vibration which is also demonstrated in Fig. 6. The amplitude of the manipulator at roller-supported end as well as the terminal end is 
significantly affected by the change in actuator frequency $\left(\Omega_{\mathrm{a}}\right)$. However, in case of rotary joint frequency $\left(\Omega_{\mathrm{J}}\right)$ even the fundamental eigenfrequency $\left(\bar{\lambda}_{1}\right)$ shifts to a value corresponding to $\bar{\lambda}_{2}$ that also leads to a shift in modes shapes as shown in Fig. 6 . In addition, it has also been noticed that, as we increase the joint frequency $\left(\Omega_{\mathrm{J}}\right)$ beyond 1.5 , system again tends to vibrate at a lower mode of vibration. A similar phenomenon has also been observed previously [18] in the case of flexible manipulator driven only by revolute joints. The presented results furnish the vital information regarding modal parameters which shall enable the identification of resonance conditions when operating frequency becomes equal or nearly equal to the system natural frequency and hence, facilitate the proper attenuation of system to avoid the inadmissible vibrations.

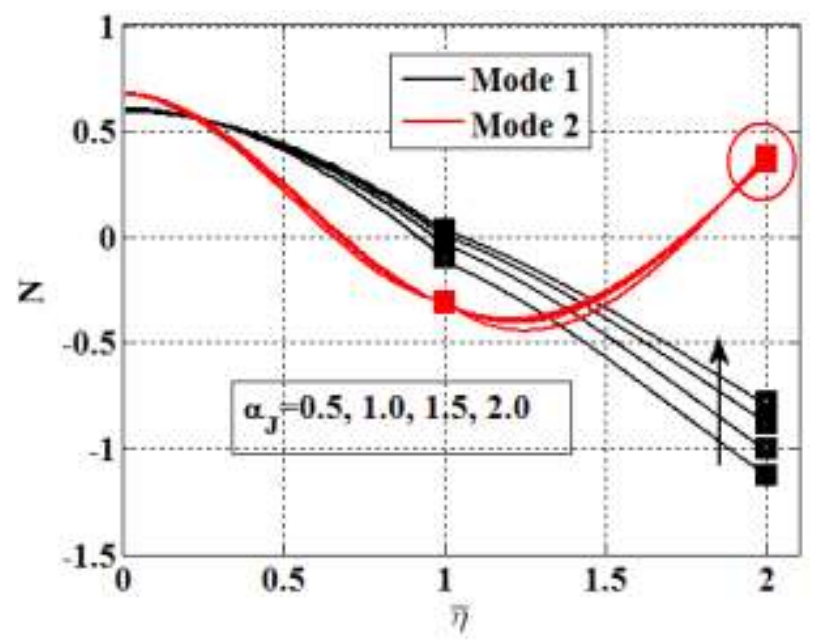

Fig. 5: Influence of joint inertia parameter $\left(\alpha_{\mathrm{J}}\right)$ on mode shapes of flexible two-link manipulator with prismatic and revolute joints with $\alpha_{\mathrm{m} 1,2, \mathrm{a}}=1.0, \chi=1.0, \alpha_{\mathrm{M}}=1.0, \Omega_{\mathrm{a}, \mathrm{J}}=0.5$.
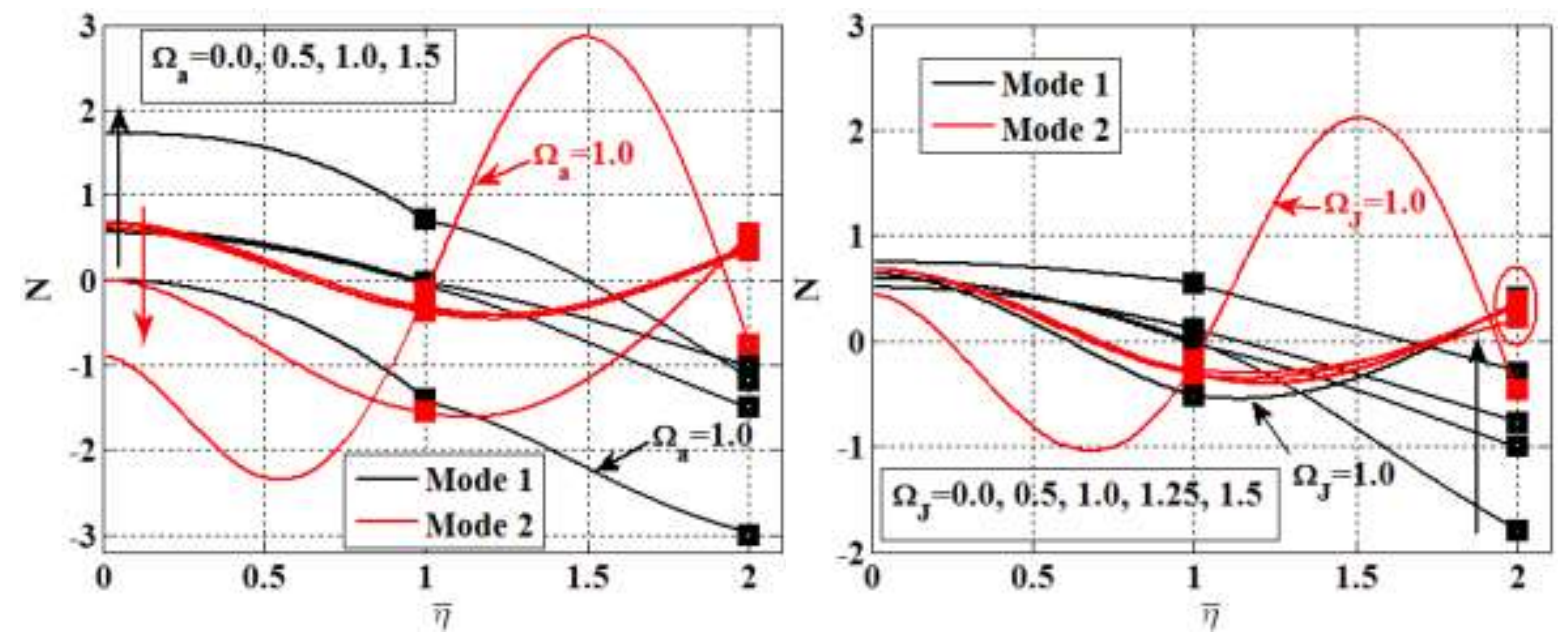

Fig. 6: Influence of actuator frequency $\left(\Omega_{\mathrm{a}}\right)$ and joint frequency parameter $\left(\Omega_{\mathrm{J}}\right)$ on mode shapes of flexible two-link manipulator with prismatic and revolute joints with $\alpha_{\mathrm{m} 1,2, \mathrm{a}}=1.0, \chi=1.0$, $\alpha_{\mathrm{M}}=1.0, \alpha_{\mathrm{J}}=1.0$. 


\subsection{Dynamic characteristics: system responses}

A computationally efficient dynamic model is obtained by discretizing the governing equations of motion of links, joints and actuator given in Eqs. (3), (4), (5) and (7). The prismatic actuator has been provided a sinusoidal force of amplitude $0.1 \mathrm{~N}$ and having duty cycle of $0.5 \mathrm{sec}$. A similar torque profile of amplitude $0.5 \mathrm{~N}-\mathrm{m}$ has been imparted to the joint for analyzing the system responses under parametric influences. In present analysis, the flexible links are considered with width, height and length as $\mathrm{b}_{1,2}=0.03 \mathrm{~m}, \mathrm{~h}_{1,2}=0.003 \mathrm{~m}$, and $\mathrm{L}_{1,2}=0.3 \mathrm{~m}$, respectively. The material of links are assumed to have mass density and Young's modulus of $\rho=7800 \mathrm{Kg} / \mathrm{m}^{3}$, and $\mathrm{E}=2.1 \times 10^{11} \mathrm{~N} / \mathrm{m}^{2}$, respectively. The other parameters are considered as; mass of joint at the end of the first link $\mathrm{m}_{1}=0.1 \mathrm{Kg}$, payload mass $\mathrm{m}_{2}=0.1 \mathrm{Kg}$, inertia of joint $\mathrm{J}=0.01 \mathrm{Kg}-\mathrm{m}^{2}$, actuator mass $\mathrm{m}_{\mathrm{a}}=0.1 \mathrm{Kg}$.
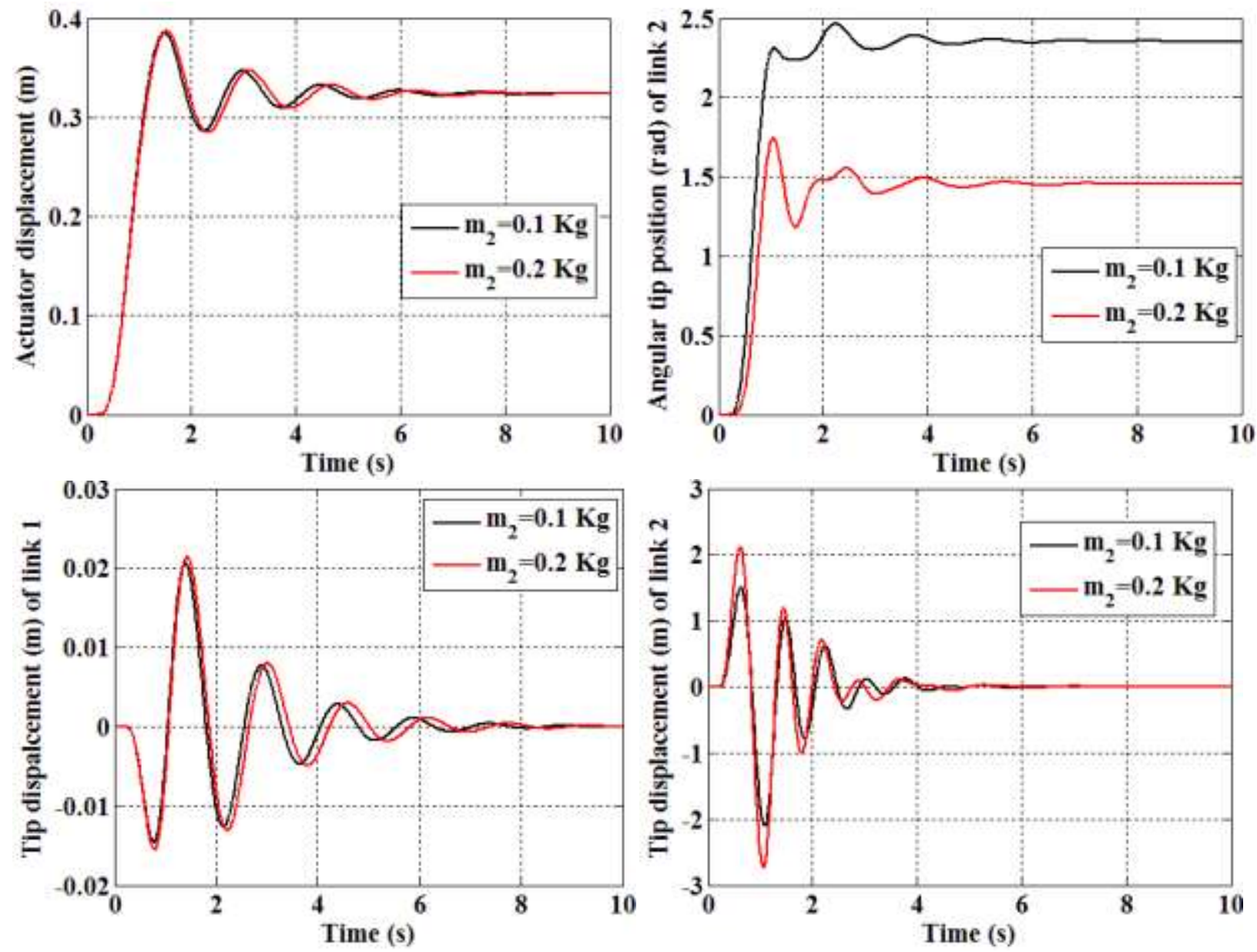

Fig 7: Influence of payload mass $\left(\alpha_{\mathrm{m} 2}\right)$ on the actuator displacement, angular tip position of link 2 , and tip displacements.

The final position of the actuator remains unchanged at $0.32 \mathrm{~m}$ with the change in payload mass as evident in Fig. 7. It can also be noticed that the angular position of link-2 decreases from 2.4 radians to 1.45 radians with increase in payload from $0.1 \mathrm{Kg}$ to $0.2 \mathrm{Kg}$, respectively. It can also be noticed that last link vibrates with reasonably larger amplitude as compared to the first link in response to the larger payload. In Fig. 8, we examine the influence of payload on the system 
responses when only prismatic joint is actuated while no input torque is provided at the revolute joint. No significant change in the actuator position is noticed but the response of the second link drastically varies if compared with the Fig. 7. If we compare the results in Figs. 7-8, it is apparent that the angular tip position of second link for payload mass of $0.1 \mathrm{~kg}$ is $2.4 \mathrm{rad}$ and $0.79 \mathrm{rad}$ for the manipulator with actuated and unactuated revolute joint, respectively. Here also, residual vibration amplitude increases with increase in payload mass. The influence of actuator mass is significant on the position of actuator as well as angular position of second link which is also shown in Fig. 9. It is visible that the actuator position decreases and the angular position of the second link increases, with increase in actuator mass. It is also noticeable in the figure that the tip of both the links vibrates at smaller amplitude for larger actuator mass. In Fig. 10, it can be observed that the settling time of actuator increases with increase in joint inertia without affecting the actuator position. However, the angular position of the second link significantly decreases and tip vibrations increase with increase in joint inertia.
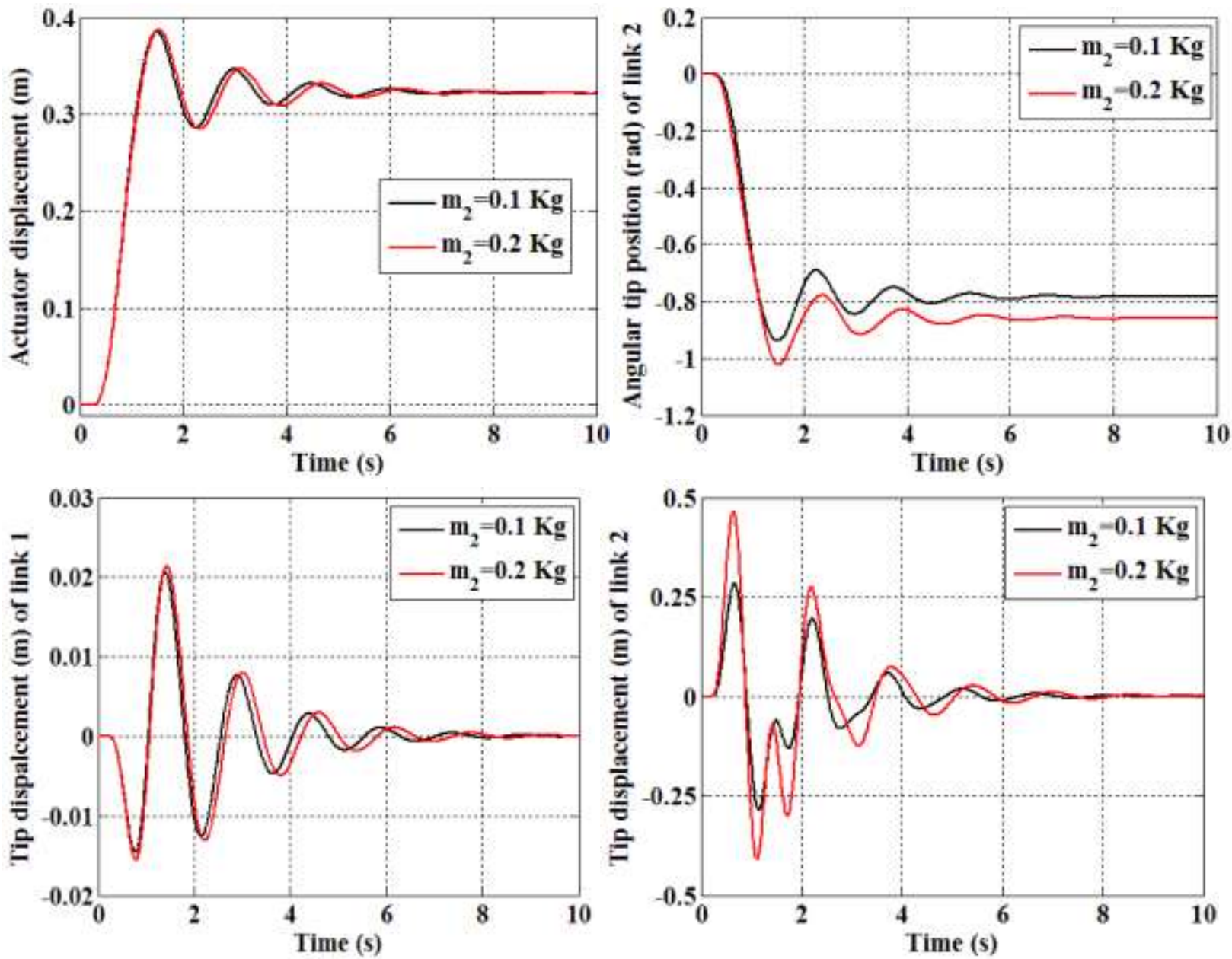

Fig 8: Influence of payload mass $\left(\mathrm{m}_{2}\right)$ on the actuator displacement, angular tip position of link 2 , and tip displacements without joint torque. 

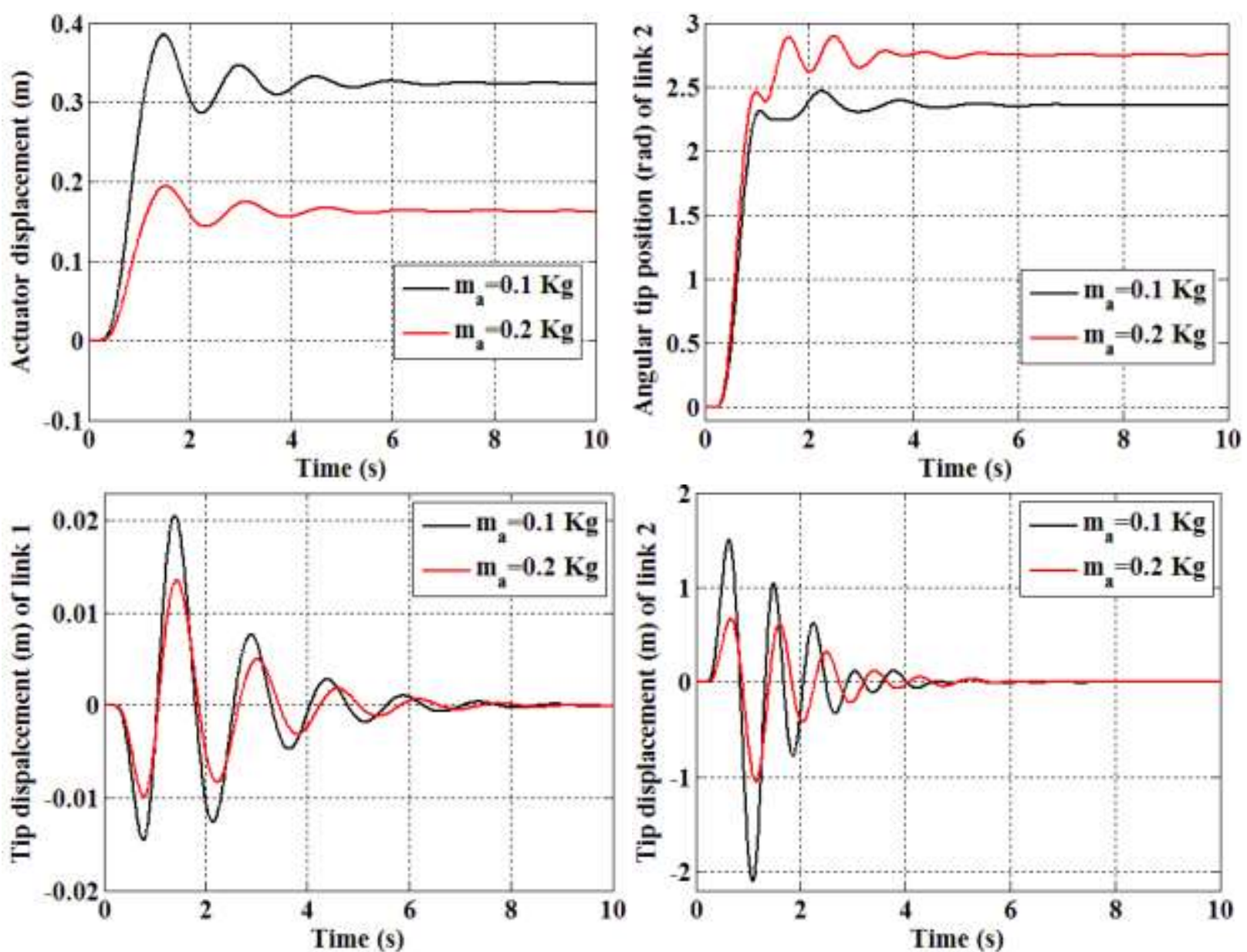

Fig 9: Influence of actuator mass $\left(\mathrm{m}_{\mathrm{a}}\right)$ on the actuator displacement, angular tip position of link 2 , and tip displacements.
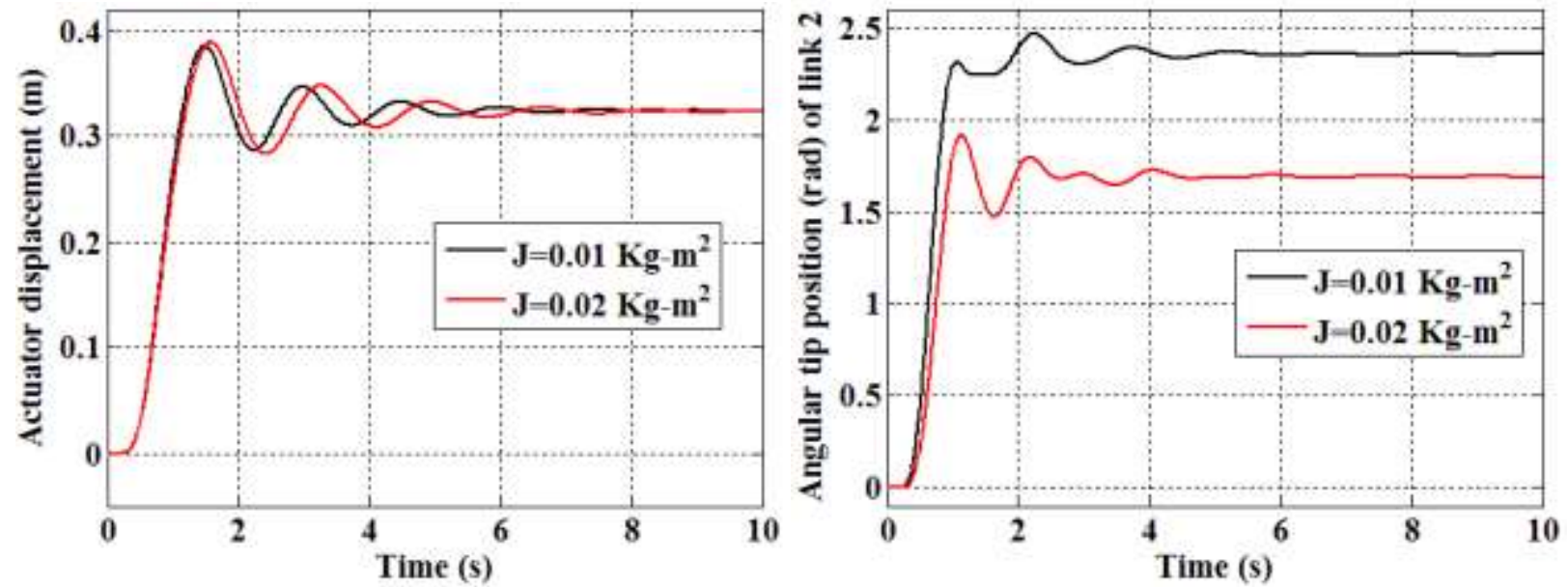

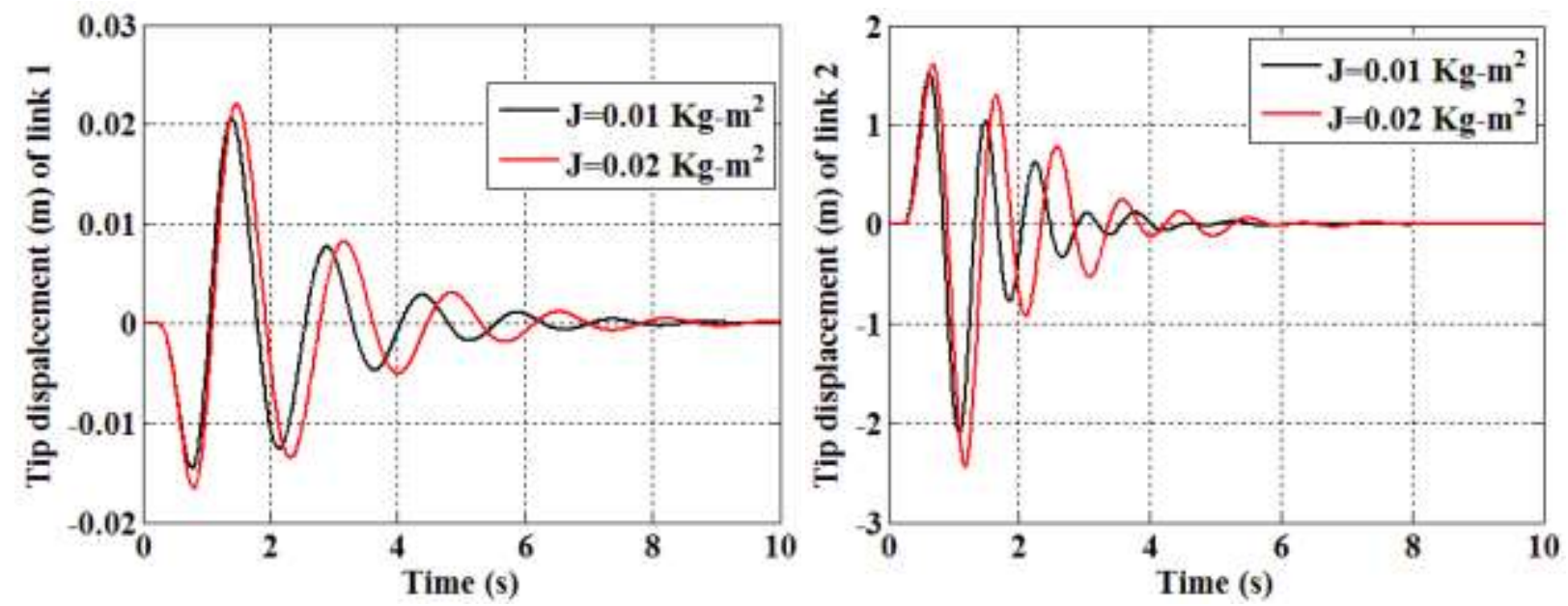

Fig 10: Influence of joint inertia $(\mathrm{J})$ on the actuator displacement, angular tip position of link 2, and tip displacements.

\subsection{Nonlinear analysis: primary and internal resonance}

In the literature, the nonlinear behavior of single-link manipulator with prismatic joint has been extensively studied [12-13] and research has been confined to the development of control strategies of flexible two-link manipulator. When the manipulator is subjected to a prismatic motion, the system experiences a forced vibration and undergoes relatively large vibrations causing undesirable vibrations due to its low stiffness which is a major concern. The adequate familiarity with the manipulator behavior under parametric variation of system attributes enables the operator to avoid the catastrophic failure under such circumstances. For primary resonance condition in both the links, the internal resonance has been avoided by considering the length ratio $\left(\alpha_{L}\right)$ of 1.1. While for internal resonance in second link the links are of same length i.e. $\alpha_{\mathrm{L}}=1.0$. The amplitude ratio for prismatic motion $\left(\bar{\vartheta}_{0}\right)$ and revolute joint motion $\bar{\theta}_{0}$ are chosen as 0.0005 and nondimensional damping coefficients $\left(\mu_{1,2}\right)$ are taken as 0.01 . In all numerical simulations, book-keeping parameter $(\varepsilon)$ and scaling factor (h) are selected as 0.1 and 0.005 , respectively.

The frequency response curves representing the amplitude ( $r$ ) of manipulator with respect to the respective motor speeds $(\sigma)$ obtained for the steady state conditions (37-40) of primary resonance cases in both these links are shown in Figs. 11-19. While the solid lines represent the stable solutions, the dashed lines depict the unstable solutions in all figures. The representative frequency response curves of first and second link for the primary resonance due to the harmonic motion imparted to the prismatic and revolute joints are shown in Fig. 11. The presence of geometric nonlinearities induces the multivalued solutions and jump phenomenon in the frequency response curves of both the links. It is observed that while the first link exhibits the spring hardening behavior with the bending of curve towards right, the second link illustrates the spring softening behavior with bending towards left. In case of first link the jump down phenomenon is observed at point $\mathrm{D}$ while increasing the motor speed and a sudden jump in amplitude of vibration is witnessed at point $G$ as the motor speed is decreased. The numerical and the corresponding analytical time response, phase portrait and FFTs have been presented in Fig. 12 and results are found to be in good agreement. 

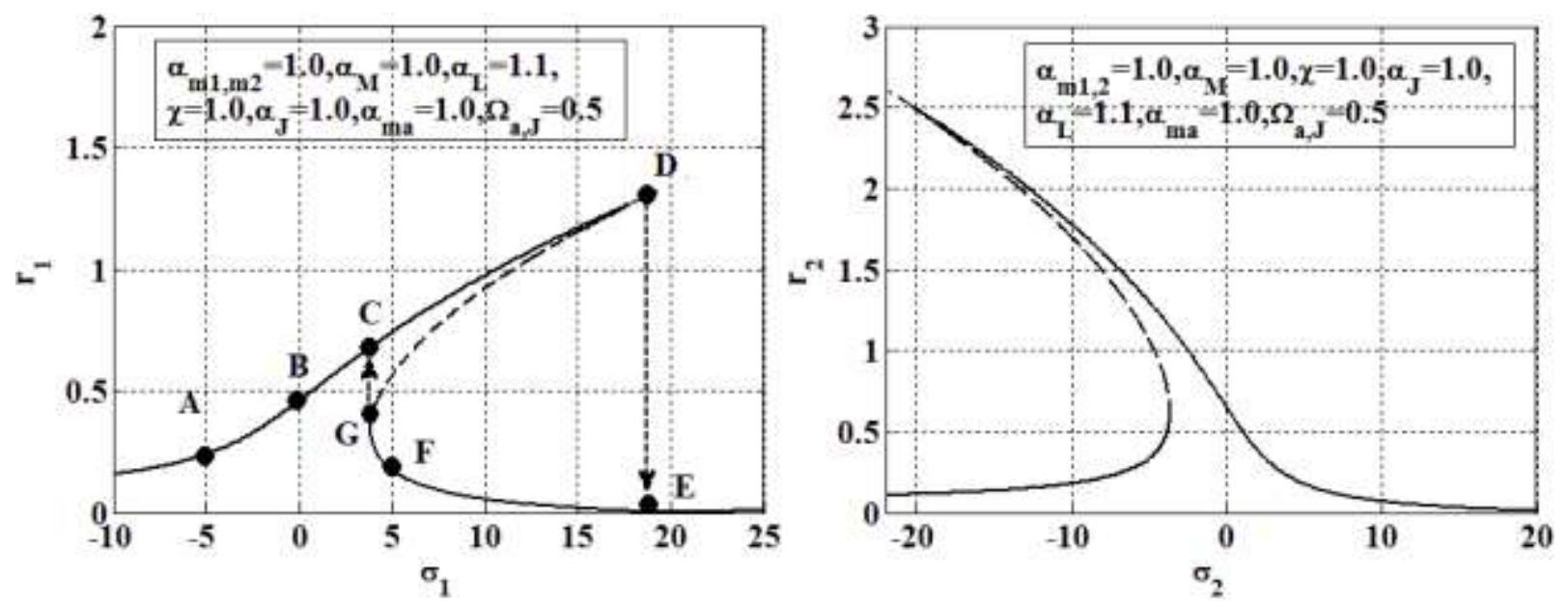

Fig. 11: Frequency response curve of first and second link of flexible two-link manipulator with prismatic and revolute joints for primary resonance case.
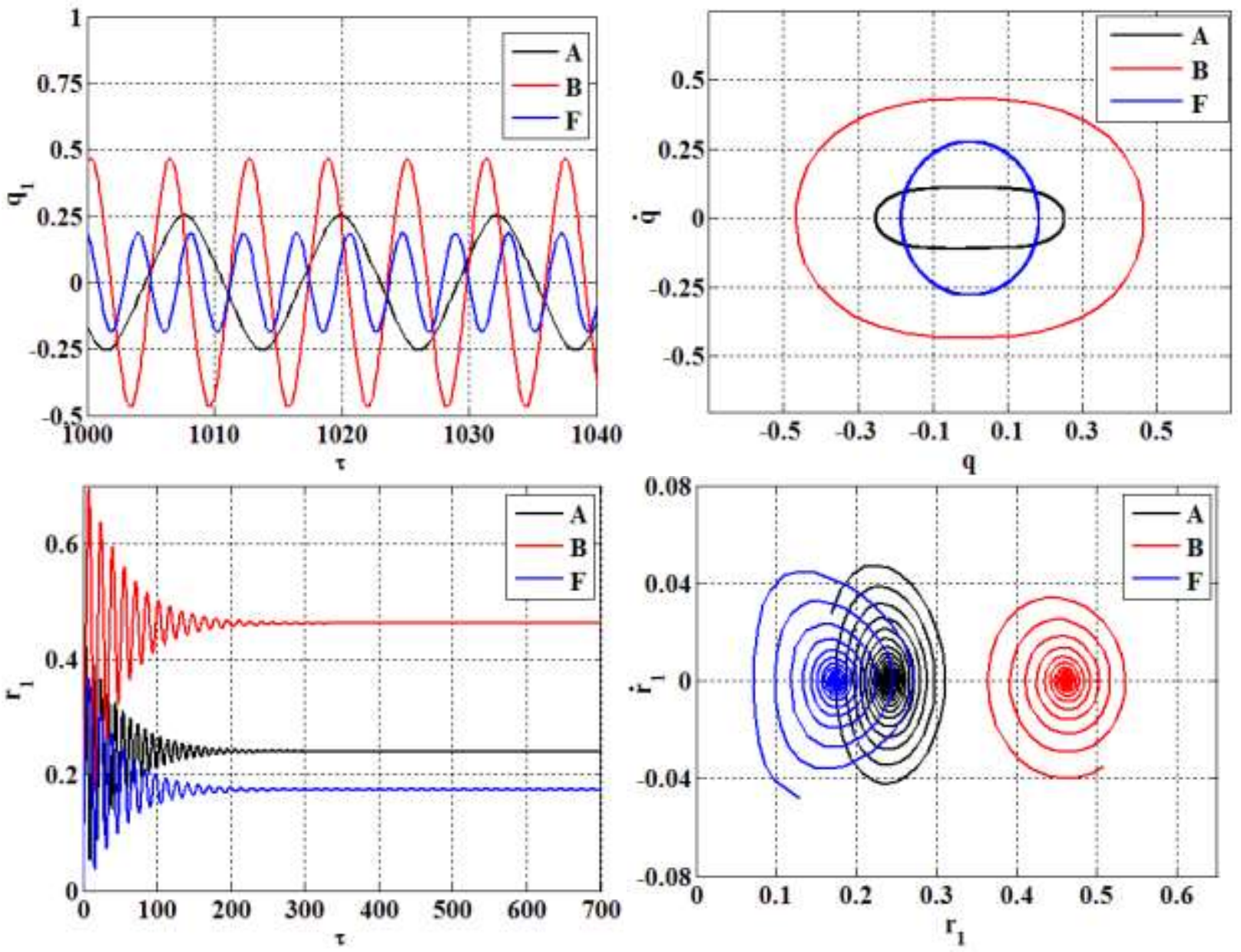


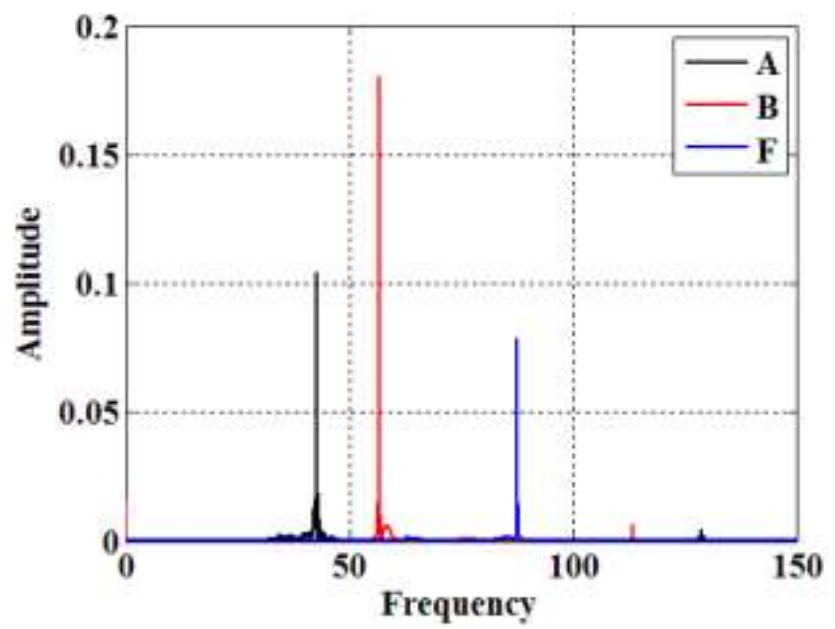

Fig 12: Numerical and analytical time history, phase portrait, and FFT at critical points A, B, and F keyed to Fig. 11.

The variation in responses of flexible links have been accomplished by investigating the influence of various dynamic factors like payload mass parameter $\left(\alpha_{\mathrm{m} 2}\right)$, flexural rigidity ratio $(\chi)$ and beam mass density $\left(\alpha_{M}\right)$ those have been controlled with cross-sectional area, joint inertia $\left(\alpha_{\mathrm{J}}\right)$ and stiffness $\left(\mathrm{k}_{\mathrm{a}, \theta}\right)$ etc. The effect of payload mass $\left(\alpha_{\mathrm{m} 2}\right)$, actuator mass $\left(\alpha_{\mathrm{ma}}\right)$, and beam mass density ratio $\left(\alpha_{M}\right)$ on the vibration responses of first link has been observed to be negligible which is evident from Fig. 13-14. However, the vibration amplitude of second link increases with increase in payload mass $\left(\alpha_{\mathrm{m} 2}\right)$, actuator mass $\left(\alpha_{\mathrm{ma}}\right)$ and beam mass density ratio $\left(\alpha_{M}\right)$. From Fig. 14, it has been noticed that change in beam density may influence the overall end-effector position significantly. Maximum vibration amplitude has been substantially increased from 2 to 3 with increase in beam density from 0.5 to 1.0. The vibration amplitude can even increase upto $50 \%$ of initial value with a slight increase in beam density. With increase in beam density, jump length i.e., sudden change in amplitude due to jump phenomena, increases substantially as compared to actuator's mass effect. However, jump length in case of jump-down phenomena is comparatively higher than that of occurred during jump-up phenomena. Due to this sudden change into large vibration oscillation, system may undergo catastrophic structural damages. Thus appropriate selection of mass density and actuator mass can offer insignificant vibration oscillation and persuade a safe operating condition. The influence of flexural rigidity ratio on the frequency response characteristics is shown in Fig. 15 and it is visible that as the flexibility ratio $(\chi)$ increases vibration amplitude of the first link decreases initially and then experiences a marginal change with further increase in flexural rigidity $\operatorname{ratio}(\chi)$. However, vibration of second link is significantly influenced by the changes in flexural rigidity. With increase in flexural rigidity, vibration amplitude increases remarkably. For a slight increase in flexural rigidity, mechanical vibration may increase approximately double of the initial state. As a result, jump length increases in a similar order as amplitude of oscillation. Hence, catastrophic failure primarily may be observed in second link due to its severe vibration tendencies. It is thus 
advisable to select an appropriate value of flexural rigidity for individual link in order to avoid large oscillation which may later lead to catastrophic failure.
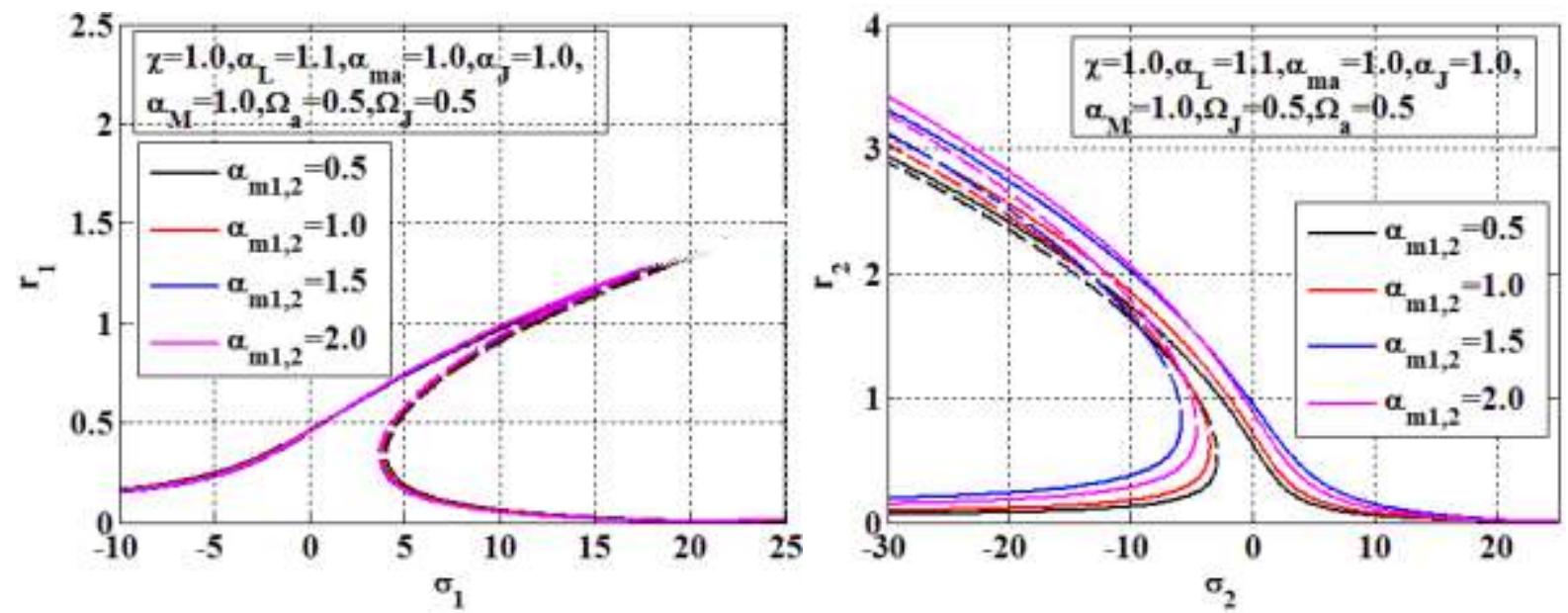

Fig. 13: Variation of frequency response curve of first and second link with of mass parameters $\left(\alpha_{\mathrm{m} 1,2}\right)$.
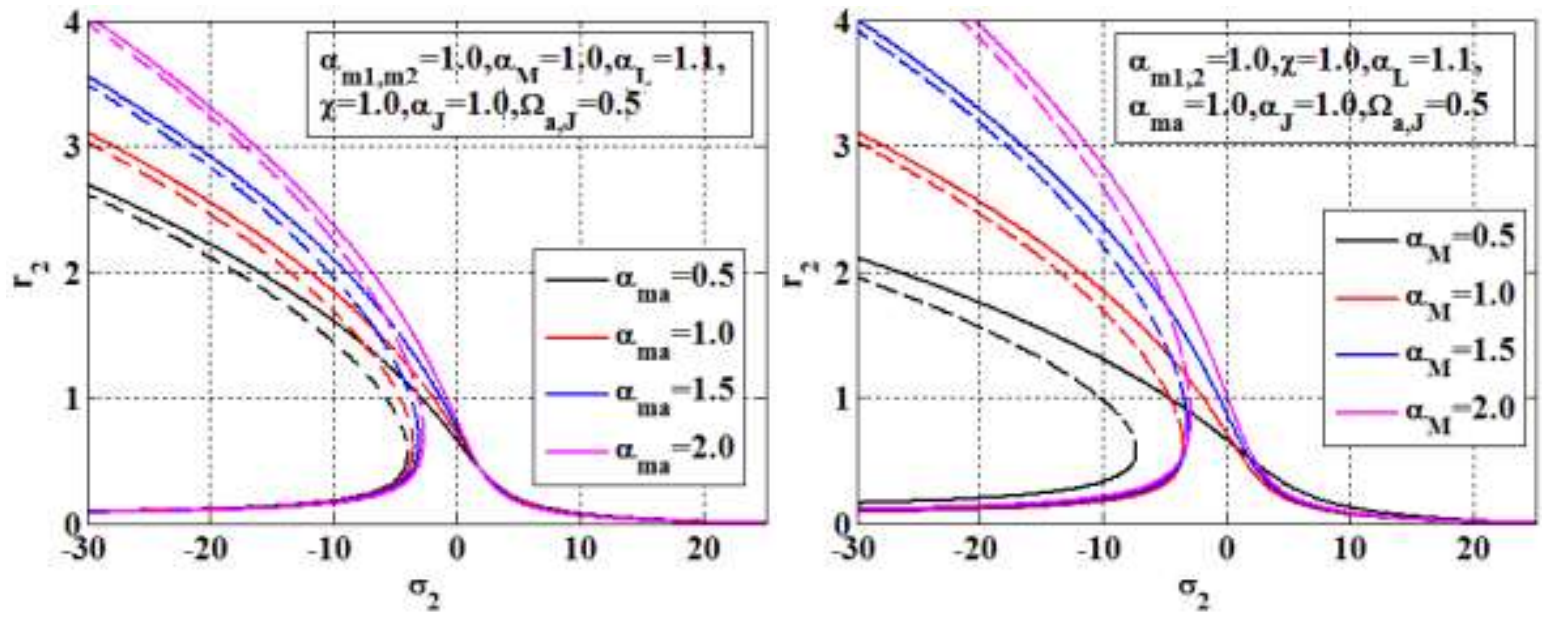

Fig. 14: Variation of frequency response curve of second link with of actuator mass parameter $\left(\alpha_{\text {ma }}\right)$ and beam mass density ratio $\left(\alpha_{M}\right)$

Figure 16 describes the effect of joint inertia on both the links and with increase in joint inertia, the amplitude of oscillation increases for the second link. Similarly, the variation in vibration amplitude has found to be insignificant for link-1 with increase in joint inertia. Thus, inappropriate selection of joint parameter can experience bifurcation and finally lead to structural damage under repetitive operations. The results presented here are in contrast with those determined in [18] for the robotic system driven by revolute joints, where the first link undergoes with an alternative vibration behavior from spring hardening to spring softening or vie-verse when parameter is subjected to alter. Thus, we can finally conclude by saying that the nonlinear system behavior is a critical function of the joint dynamics. As a result, any slight change in the configuration of joint dynamics may cause detrimental consequences on overall system dynamics. 

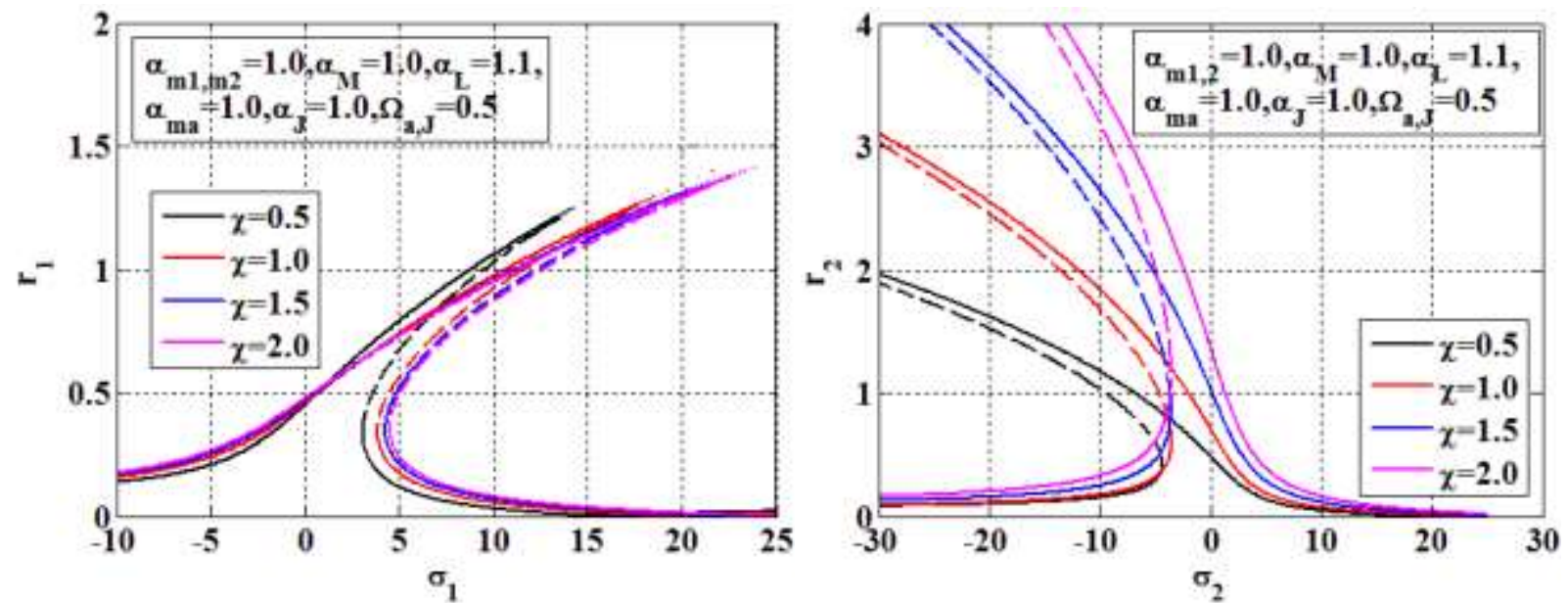

Fig. 15: Variation of frequency response curve of first and second link with flexural rigidity ratio $(\chi)$.

The actuator frequency $\left(\Omega_{\mathrm{a}}\right)$ and revolute joint frequency $\left(\Omega_{\mathrm{J}}\right)$ individually represents the actuator stiffness $\left(\mathrm{k}_{\mathrm{a}}\right)$ and torsional joint stiffness $\left(\mathrm{k}_{\theta}\right)$. From Fig. 17, the change in actuator frequency $\left(\Omega_{\mathrm{a}}\right)$ between 0 and 2.0 can observe a behavioral transformation for first link from spring hardening to spring softening. It is interesting to note that this transformation primarily occurred when the actuator frequency is equal to 1.0 and at all other values; the response is restricted to spring hardening behavior with decreasing amplitude. Due to this sudden change in actuator frequency to unit magnitude from any other non-zero value, system may demonstrate fatigue characteristics and several such changes may lead to fatigue failure. Therefore, one must note that system should not operate with an actuator frequency equal to 1.0 to avoid any fatigue failure. However, responses of flexible second link are restricted to softening behavior for all values of actuator frequencies. But, it has been observed that with increase $\Omega_{\mathrm{a}}$, amplitude of oscillation decreases except for $\Omega_{\mathrm{a}}$ equal to 1.0. When the system operates with a frequency equal to 1.0, it undergoes acute oscillation with large vibration amplitude. Thus the actuator frequency equal to 1.0 is found to be critical operating condition for both these links. This is because the sudden shift in natural frequency when actuator frequency becomes 1. A slight change in actuator frequency can raise the natural frequency to the higher value. Hence, one should avoid this situation to maintain safe and secure operating conditions. However, considering higher value of actuator frequency can enable minimum vibration characteristic.

Similarly, the influence of joint frequency on the vibration characteristics has also been studied in Fig. 17. Similar trend is also observed here that with increase in joint frequency, amplitude of vibration of link-1 decreases except for $\Omega_{\mathrm{J}}$ equal to 1 while the nature of vibration characteristics always maintains to be of hardening behavior. Vibration amplitude increases to its maximum value when the system is subjected to $\Omega_{\mathrm{J}}$ equals to 1.0. In contrary, with increase in joint frequency, for $\Omega_{\mathrm{J}}<1.0$, response amplitude of link-2 increases while it is exactly opposite for $1.0<\Omega_{\mathrm{J}}<1.5$ where vibration amplitude gets decreases significantly. It is interesting to note that for any value of $\Omega_{\mathrm{J}}>1.0$, behavior changes to hardening from softening. Therefore, 
selection of joint frequency decides whether system exhibits hardening behavior or softening behavior or mix vibrations.
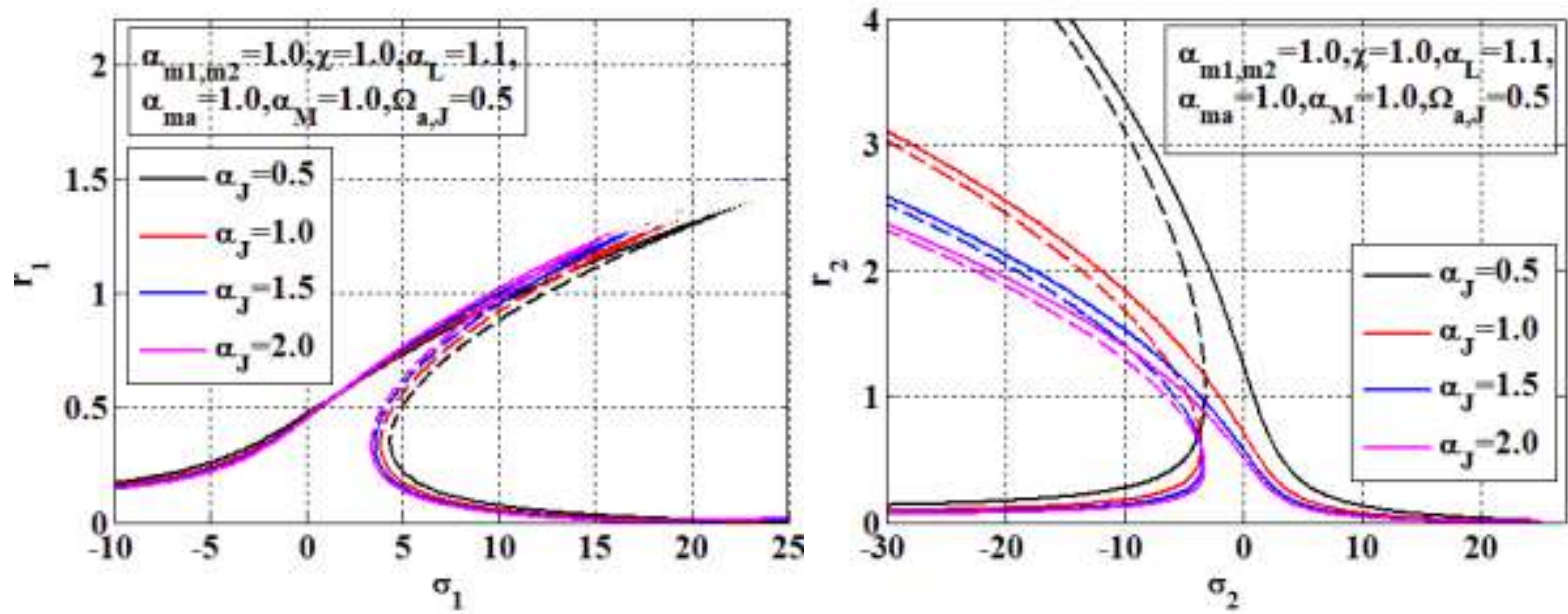

Fig. 16: Variation of frequency response curve of first and second link with joint inertia $\operatorname{parameter}\left(\alpha_{\mathrm{J}}\right)$.
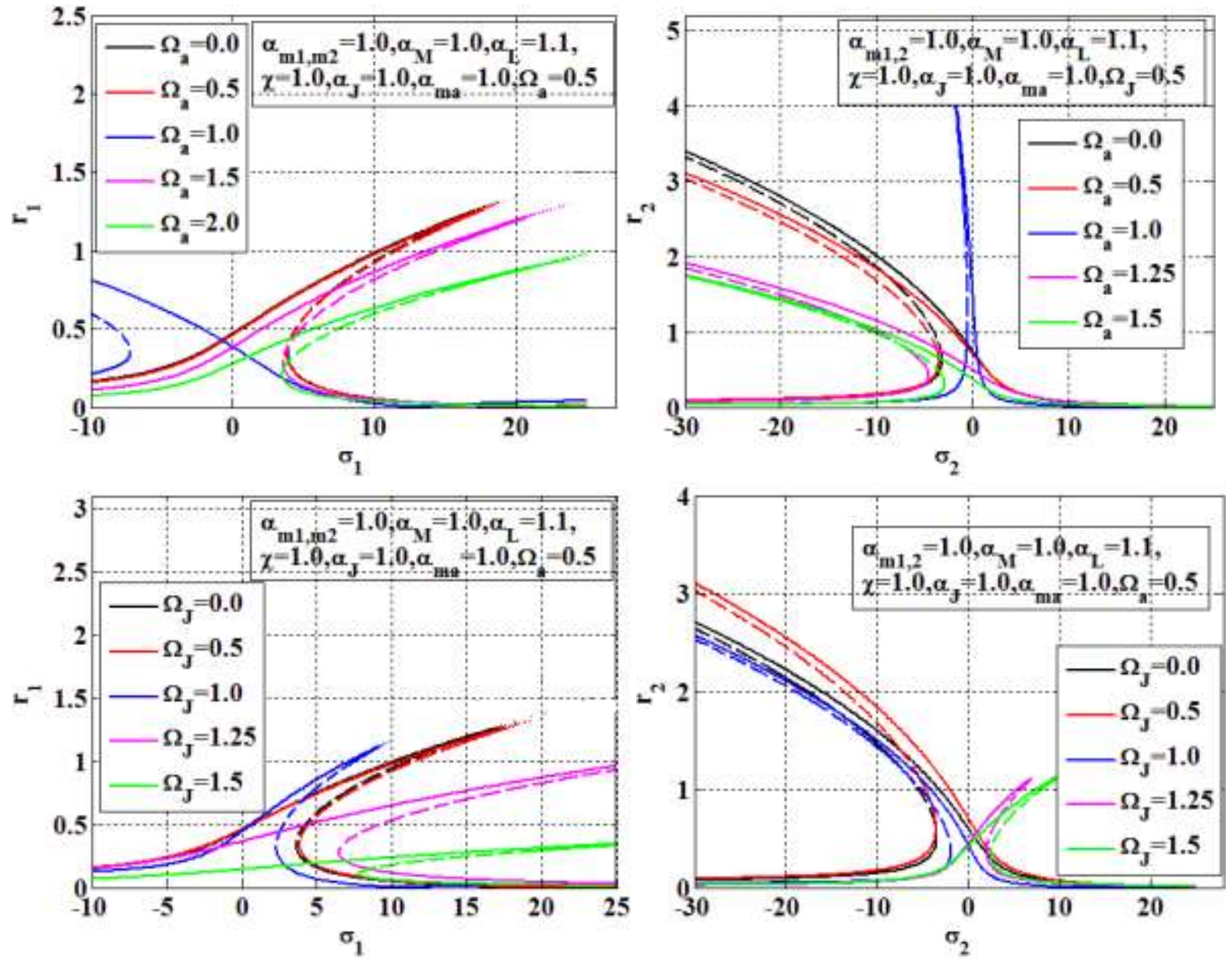

Fig. 17: Variation of frequency response curve of first and second link with actuator frequency $\operatorname{parameter}\left(\Omega_{\mathrm{a}}\right)$ and joint frequency parameter $\left(\Omega_{\mathrm{J}}\right)$. 
Figure 18 shows the behavioral differences between primary resonance condition and internal resonance condition in the second link. However, resonant behaviors found in these two resonance conditions are quite similar in nature and observed to be softening behavior. The response amplitude in internal resonance condition is found to be higher than that of primary resonance condition for the same values of design parameters. Primary resonance condition acts as a forced vibration phenomenon in the second link and synchronization of these two resonance conditions instigate the severity of vibration in the second link.
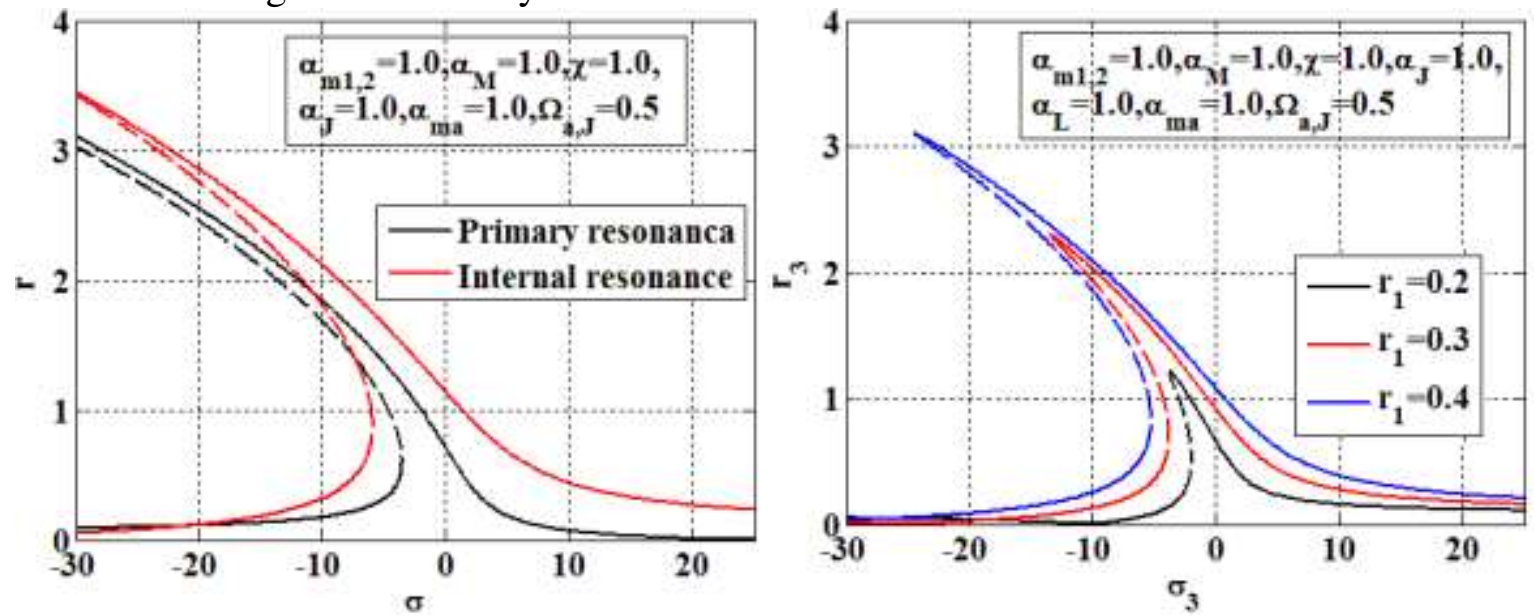

Fig. 18: Comparison of frequency response curve of second link for primary and internal resonance and effect of amplitude of first link $\left(\mathrm{r}_{1}\right)$.
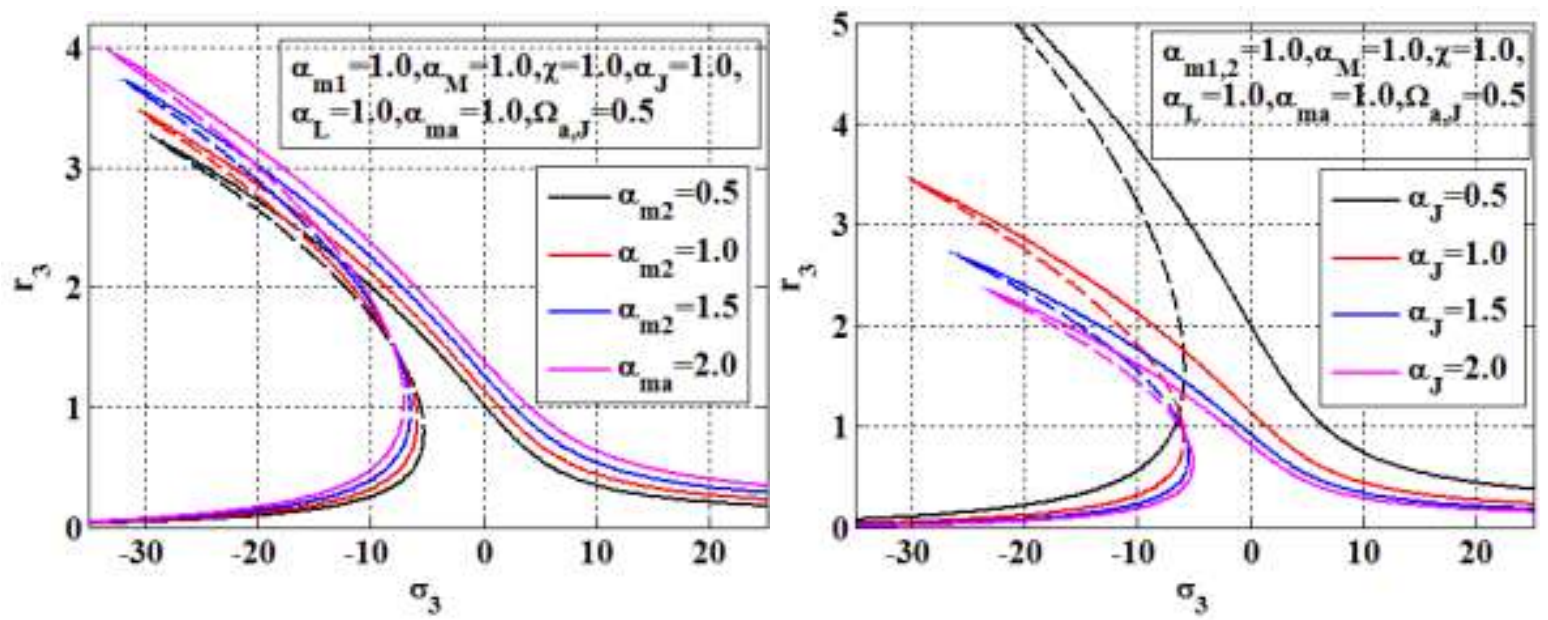

Fig. 19: Variation of frequency response curve of second link with payload mass parameter $\left(\alpha_{\mathrm{m} 2}\right)$ and joint inertia parameter $\left(\alpha_{\mathrm{J}}\right)$ for internal resonance case.

It is interesting to note that, vibration amplitude of link-1 has a significant influence on the vibration of second link. It is observed that vibration amplitude of link-1 may offer a positive impact in order to reduce the vibration level of second link. As a result, with decrease in vibration amplitude $r_{1}$, one can reduce the vibration level in second link and it behaves as a damping phenomenon in a same vibrating phase. Hence, appropriate selection of vibration parameters of link-1 may decline the possibility of vibration oscillation of link-2. Furthermore, the amplitude of the link- 2 vibration increases and decreases, respectively with increase in 
payload mass parameter $\left(\alpha_{\mathrm{m} 2}\right)$ and joint inertia $\left(\alpha_{\mathrm{J}}\right)$ parameter as illustrated in Fig. 19. Therefore, one may choose appropriate values of payload and joint parameters to keep the operating conditions into a desire lever.

The present analysis can be satisfactorily applied to the long-reach robot manipulators which are involved in most of the pick and drop operations. The derived eigenspectrums can be adopted in developing the suitable control schemes to attain desire end-effector trajectories while nonlinear analysis of manipulators undergoing large deformations may predict the actual responses under any external influences. Nowadays, the manipulators are being used in different adverse conditions where the end-effector is subjected to various forcing conditions with different joint configurations and further, end-effector may sometimes move along the link to perform various operations. The present linear and nonlinear approach can offer insightful dynamics and certain design criteria of two-links flexible manipulator having both prismatic and revolute joint.

\section{Conclusions}

In current study, an attempt has been made to explore the modal analysis for calculating eigenfrequencies and eigenspectrums further to study the tip trajectory characteristics. In addition, nonlinear stability analysis has been carried put to inhibit the local bifurcations for long-term solutions when the flexible joints have been imparted with harmonic motion. Subsequently, the influence of parametric variation of design parameters on the eigenbehaviors, tip trajectories, stability and bifurcation phenomena has been graphically demonstrated. The critical and pin-point outcomes and conclusions thus obtained are bulleted as below.

- The addition of inertia through payload mass, joint inertia, actuator mass, and beam mass decreases the system natural frequencies. The eigenfrequencies tend to increase with the addition of stiffness of the system by increasing flexural rigidity and stiffness component of flexible joints. However, eigenfrequencies witness a sudden jump to a higher mode of vibration at unit magnitude of joint frequency.

- The amplitude of roller-supported and terminal end decreases respectively with the increase of actuator and payload masses. The flexural rigidity of links significantly influences the amplitude of lower modes of vibration. At unit magnitude of the joint parameter represents the limiting condition when the joint dynamics gets decoupled from the manipulator system and system starts vibrating at a higher mode of vibration.

- The actuator mass significantly affects the actuator position as well as the angular response of the joint. The increase in payload mass and joint inertia decreases the angular position of joint without affecting the position of actuator. The joint reverses its angular position when the joint torque is removed and only actuator force is imparted.

- Second order method of multiple scales has been used to analytically investigate the system response under forcing conditions imparted though harmonically driven prismatic and revolute joints. The multi-valued solution and jump phenomenon due to static saddle-node bifurcations has been noticed. Numerically, it is established that internal resonance exists between both the links and hence, simple resonance in both the links and internal resonance in second link is investigated under the parametric variation of system attributes.

- The influence of the payload mass, actuator mass and beam's mass density ratio has been found to be negligible on the nonlinear response of first-link of the manipulator while the amplitude at a specific frequency and hence the jump length of second link increases with the payload mass, actuator mass and beam's mass density ratio. Both the links witness the 
behavioral changes into spring softening to spring hardening or vice-versa with the variation of actuator and joint frequencies. The change in sudden amplitude due to jump phenomenon and dynamic behavior may cause the catastrophic failure of the system and hence the better understanding of the parametric variation of system response is essential in order to operate the manipulator safely.

- The developed results can be successfully used to develop the control strategy and suppress the end-point vibrations of flexible two-link manipulators operating in the field of nuclear power plants, microsurgery, and space applications where one link is restrained to move in Cartesian plane and other link exhibits planar rotation. The present analysis can be extended to study the system behavior of manipulator under large deformation or the manipulators subjected to various forcing conditions simulating the environment in which the system is supposed to operate.

\section{Appendix}

The elements of the matrix in Eq. (15) are expressed below:

$$
\begin{aligned}
& \mathrm{U}_{11}^{\mathrm{m}}=-\alpha_{\mathrm{ma}} \bar{\lambda}^{\mathrm{m}}\left(1-\Omega_{\mathrm{a}}^{2}\right), \mathrm{U}_{12}^{\mathrm{m}}=-2, \mathrm{U}_{13}^{\mathrm{m}}=-\alpha_{\mathrm{ma}} \bar{\lambda}^{\mathrm{m}}\left(1-\Omega_{\mathrm{a}}^{2}\right), \\
& \mathrm{U}_{21}^{\mathrm{m}}=-\mathrm{s}\left(\bar{\lambda}^{\mathrm{m}}\right)-\left(\alpha_{\mathrm{m} 1}+\alpha_{\mathrm{m} 2}+\alpha_{\mathrm{M}} \alpha_{\mathrm{L}}\right) \bar{\lambda}^{\mathrm{m}} \mathrm{c}\left(\bar{\lambda}^{\mathrm{m}}\right) \text {, } \\
& \mathrm{U}_{22}^{\mathrm{m}}=\mathrm{c}\left(\bar{\lambda}^{\mathrm{m}}\right)+\operatorname{ch}\left(\bar{\lambda}^{\mathrm{m}}\right)-\left(\alpha_{\mathrm{m} 1}+\alpha_{\mathrm{m} 2}+\alpha_{\mathrm{M}} \alpha_{\mathrm{L}}\right) \bar{\lambda}^{\mathrm{m}}\left\{\mathrm{s}\left(\bar{\lambda}^{\mathrm{m}}\right)-\operatorname{sh}\left(\bar{\lambda}^{\mathrm{m}}\right)\right\} \text {, } \\
& \mathrm{U}_{23}^{\mathrm{m}}=-\operatorname{sh}\left(\bar{\lambda}^{\mathrm{m}}\right)-\left(\alpha_{\mathrm{m} 1}+\alpha_{\mathrm{m} 2}+\alpha_{\mathrm{M}} \alpha_{\mathrm{L}}\right) \bar{\lambda}^{\mathrm{m}} \operatorname{ch}\left(\bar{\lambda}^{\mathrm{m}}\right), \mathrm{U}_{25}^{\mathrm{m}}=\chi \kappa^{3}, \mathrm{U}_{27}^{\mathrm{m}}=-\chi \kappa^{3}, \mathrm{U}_{31}^{\mathrm{m}}=-\mathrm{c}\left(\bar{\lambda}^{\mathrm{m}}\right) \text {, } \\
& \mathrm{U}_{32}^{\mathrm{m}}=-\mathrm{s}\left(\bar{\lambda}^{\mathrm{m}}\right)-\operatorname{sh}\left(\bar{\lambda}^{\mathrm{m}}\right), \mathrm{U}_{33}^{\mathrm{m}}=\operatorname{ch}\left(\bar{\lambda}^{\mathrm{m}}\right), \mathrm{U}_{34}^{\mathrm{m}}=\chi \kappa^{2}, \mathrm{U}_{36}^{\mathrm{m}}=-\chi \kappa^{2}, \mathrm{U}_{41}^{\mathrm{m}}=\mathrm{c}\left(\bar{\lambda}^{\mathrm{m}}\right) \text {, } \\
& \mathrm{U}_{42}^{\mathrm{m}}=\mathrm{s}\left(\bar{\lambda}^{\mathrm{m}}\right)-\operatorname{sh}\left(\bar{\lambda}^{\mathrm{m}}\right), \mathrm{U}_{43}^{\mathrm{m}}=\operatorname{ch}\left(\bar{\lambda}^{\mathrm{m}}\right), \mathrm{U}_{44}^{\mathrm{m}}=-\chi, \mathrm{U}_{46}^{\mathrm{m}}=-\chi, \\
& \mathrm{U}_{51}^{\mathrm{m}}=\alpha_{\mathrm{J}}\left(1-\Omega_{\mathrm{J}}^{2}\right) \bar{\lambda}^{\mathrm{m}} \mathrm{s}\left(\bar{\lambda}^{\mathrm{m}}\right), \mathrm{U}_{52}^{\mathrm{m}}=-\alpha_{\mathrm{J}}\left(1-\Omega_{\mathrm{J}}^{2}\right) \bar{\lambda}^{\mathrm{m}}\left\{\mathrm{c}\left(\bar{\lambda}^{\mathrm{m}}\right)-\operatorname{ch}\left(\bar{\lambda}^{\mathrm{m}}\right)\right\}, \\
& \mathrm{U}_{53}^{\mathrm{m}}=-\alpha_{\mathrm{J}}\left(1-\Omega_{\mathrm{J}}^{2}\right) \bar{\lambda}^{\mathrm{m}} \operatorname{sh}\left(\bar{\lambda}^{\mathrm{m}}\right) \text {, } \\
& \mathrm{U}_{54}^{\mathrm{m}}=\left\{\alpha_{\mathrm{M}} \chi /\left(\kappa \bar{\lambda}^{\mathrm{m}}\right)^{2}\right\}\left\{\mathrm{c}\left(\gamma \bar{\lambda}^{\mathrm{m}}\right)+\kappa \bar{\lambda}^{\mathrm{m}} \alpha_{\mathrm{L}} \mathrm{s}\left(\gamma \bar{\lambda}^{\mathrm{m}}\right)-1\right\}+\alpha_{\mathrm{m} 2} \alpha_{\mathrm{L}} \chi \mathrm{c}\left(\gamma \bar{\lambda}^{\mathrm{m}}\right), \\
& \mathrm{U}_{55}^{\mathrm{m}}=\alpha_{\mathrm{J}} \bar{\lambda}^{\mathrm{m}} \chi \kappa\left(1-\Omega_{\mathrm{J}}^{2}\right)+\left\{\alpha_{\mathrm{M}} \chi /\left(\kappa \bar{\lambda}^{\mathrm{m}}\right)^{2}\right\}\left\{\mathrm{s}\left(\gamma \bar{\lambda}^{\mathrm{m}}\right)-\kappa \bar{\lambda}^{\mathrm{m}} \alpha_{\mathrm{L}} \mathrm{c}\left(\gamma \bar{\lambda}^{\mathrm{m}}\right)\right\}+\alpha_{\mathrm{m} 2} \alpha_{\mathrm{L}} \chi \mathrm{s}\left(\gamma \bar{\lambda}^{\mathrm{m}}\right), \\
& \mathrm{U}_{56}^{\mathrm{m}}=\left\{\alpha_{\mathrm{m}} \chi /\left(\kappa \bar{\lambda}^{\mathrm{m}}\right)^{2}\right\}\left\{\kappa \bar{\lambda}^{\mathrm{m}} \alpha_{\mathrm{L}} \operatorname{sh}\left(\gamma \bar{\lambda}^{\mathrm{m}}\right)-\operatorname{ch}\left(\gamma \bar{\lambda}^{\mathrm{m}}\right)+1\right\}+\alpha_{\mathrm{m} 2} \alpha_{\mathrm{L}} \chi \operatorname{ch}\left(\gamma \bar{\lambda}^{\mathrm{m}}\right) \text {, } \\
& \mathrm{U}_{57}^{\mathrm{m}}=\alpha_{\mathrm{J}} \bar{\lambda}^{\mathrm{m}} \kappa \chi\left(1-\Omega^{2}{ }_{\mathrm{J}}\right)+\left\{\alpha_{\mathrm{M}} \chi /\left(\kappa \bar{\lambda}^{\mathrm{m}}\right)^{2}\right\}\left\{\kappa \bar{\lambda}^{\mathrm{m}} \alpha_{\mathrm{L}} \operatorname{ch}\left(\gamma \bar{\lambda}^{\mathrm{m}}\right)-\operatorname{sh}\left(\gamma \bar{\lambda}^{\mathrm{m}}\right)\right\}+\alpha_{\mathrm{m} 2} \alpha_{\mathrm{L}} \chi \operatorname{sh}\left(\gamma \bar{\lambda}^{\mathrm{m}}\right), \\
& \mathrm{U}_{64}^{\mathrm{m}}=\mathrm{c}\left(\gamma \bar{\lambda}^{\mathrm{m}}\right), \mathrm{U}_{65}^{\mathrm{m}}=\mathrm{s}\left(\gamma \bar{\lambda}^{\mathrm{m}}\right), \mathrm{U}_{66}^{\mathrm{m}}=-\operatorname{ch}\left(\gamma \bar{\lambda}^{\mathrm{m}}\right), \mathrm{U}_{67}^{\mathrm{m}}=-\operatorname{sh}\left(\gamma \bar{\lambda}^{\mathrm{m}}\right), \\
& \mathrm{U}_{74}^{\mathrm{m}}=\alpha_{\mathrm{m} 2} \bar{\lambda}^{\mathrm{m}} \mathrm{c}\left(\gamma \bar{\lambda}^{\mathrm{m}}\right)+\chi \kappa^{3} \mathrm{~s}\left(\gamma \bar{\lambda}^{\mathrm{m}}\right), \mathrm{U}_{75}^{\mathrm{m}}=\alpha_{\mathrm{m} 2} \bar{\lambda}^{\mathrm{m}} \mathrm{s}\left(\gamma \bar{\lambda}^{\mathrm{m}}\right)-\chi \kappa^{3} \mathrm{c}\left(\gamma \bar{\lambda}^{\mathrm{m}}\right), \\
& \mathrm{U}_{76}^{\mathrm{m}}=\alpha_{\mathrm{m} 2} \bar{\lambda}^{\mathrm{m}} \operatorname{ch}\left(\gamma \bar{\lambda}^{\mathrm{m}}\right)+\chi \kappa^{3} \operatorname{sh}\left(\gamma \bar{\lambda}^{\mathrm{m}}\right), \quad \mathrm{U}_{77}^{\mathrm{m}}=\alpha_{\mathrm{m} 2} \bar{\lambda}^{\mathrm{m}} \operatorname{sh}\left(\gamma \bar{\lambda}^{\mathrm{m}}\right)+\chi \kappa^{3} \operatorname{ch}\left(\gamma \bar{\lambda}^{\mathrm{m}}\right), \\
& \mathrm{U}_{14}^{\mathrm{m}}, \mathrm{U}_{15}^{\mathrm{m}}, \mathrm{U}_{16}^{\mathrm{m}}, \mathrm{U}_{17}^{\mathrm{m}}, \mathrm{U}_{24}^{\mathrm{m}}, \mathrm{U}_{26}^{\mathrm{m}}, \mathrm{U}_{35}^{\mathrm{m}}, \mathrm{U}_{37}^{\mathrm{m}}, \mathrm{U}_{45}^{\mathrm{m}}, \mathrm{U}_{47}^{\mathrm{m}}, \mathrm{U}_{61}^{\mathrm{m}}, \mathrm{U}_{62}^{\mathrm{m}}, \mathrm{U}_{63}^{\mathrm{m}}, \mathrm{U}_{71}^{\mathrm{m}}, \mathrm{U}_{72}^{\mathrm{m}} \text {, and } \mathrm{U}_{73}^{\mathrm{m}}=0 \text {. }
\end{aligned}
$$

The non-dimensional parameters appearing in above equations are: 


$$
\begin{aligned}
& \overline{\mathrm{n}}=\mathrm{n} / \mathrm{L}_{1}, \bar{\lambda}=\lambda \mathrm{L}_{1}, \chi=\mathrm{E}_{2} \mathrm{I}_{2} / \mathrm{E}_{1} \mathrm{I}_{1}, \lambda^{4}=\rho_{1} \mathrm{~A}_{1} \omega^{2} / \mathrm{E}_{1} \mathrm{I}_{1}, \alpha_{\mathrm{M}}=\rho_{2} \mathrm{~A}_{2} / \rho_{1} \mathrm{~A}_{1}, \kappa^{4}=\alpha_{\mathrm{M}} / \chi, \alpha_{\mathrm{L}}=\mathrm{L}_{2} / \mathrm{L}_{1}, \\
& \alpha_{\mathrm{m} 1}=\mathrm{m}_{1} / \rho_{1} \mathrm{~A}_{1} \mathrm{~L}_{1}, \alpha_{\mathrm{m} 2}=\mathrm{m}_{2} / \rho_{1} \mathrm{~A}_{1} \mathrm{~L}_{1}, \alpha_{\mathrm{J}}=\mathrm{J} / \rho_{1} \mathrm{~A}_{1} \mathrm{~L}_{1}^{3}, \Omega_{\mathrm{a}}=\left(\mathrm{k}_{\mathrm{a}} / \mathrm{m}_{\mathrm{a}}\right) / \omega^{2}, \Omega_{\mathrm{J}}=\left(\mathrm{k}_{\theta} / \mathrm{J}\right) / \omega^{2}, \text { and } \\
& \gamma=\kappa \alpha_{\mathrm{L}} .
\end{aligned}
$$

The integration constants in Eqs. (13-14) are:

$$
\begin{aligned}
& R_{2}^{\mathrm{m}}=\left\{\mathrm{U}_{53} \mathrm{U}_{11}-\mathrm{U}_{51} \mathrm{U}_{13}-\left(\begin{array}{c}
\pi_{5} \mathrm{U}_{54}+\pi_{9} \mathrm{U}_{55} \\
+\pi_{7} \mathrm{U}_{56}+\pi_{11} \mathrm{U}_{57}
\end{array}\right) \mathrm{U}_{13}\right\}^{\mathrm{m}} /\left\{\mathrm{U}_{52} \mathrm{U}_{13}-\mathrm{U}_{53} \mathrm{U}_{12}+\left(\begin{array}{l}
\pi_{6} \mathrm{U}_{54}+\pi_{10} \mathrm{U}_{55} \\
+\pi_{8} \mathrm{U}_{56}+\pi_{12} \mathrm{U}_{57}
\end{array}\right) \mathrm{U}_{13}\right\}^{\mathrm{m}}, \\
& \mathrm{R}_{3}^{\mathrm{m}}=\left(-\mathrm{U}_{11}-\mathrm{R}_{2} \mathrm{U}_{12}\right)^{\mathrm{m}} / \mathrm{U}_{13}^{\mathrm{m}}, \mathrm{S}_{1}^{\mathrm{m}}=\pi_{5}+\pi_{6} \mathrm{U}_{2}^{\mathrm{m}}, \mathrm{S}_{2}^{\mathrm{m}}=\pi_{9}+\pi_{10} \mathrm{U}_{2}^{\mathrm{m}}, \mathrm{S}_{3}^{\mathrm{m}}=\pi_{7}+\pi_{8} \mathrm{U}_{2}^{\mathrm{m}}, \\
& \mathrm{S}_{4}^{\mathrm{m}}=\pi_{11}+\pi_{12} \mathrm{U}_{2}^{\mathrm{m}} .
\end{aligned}
$$

Here,

$$
\begin{aligned}
& \pi_{1}=\left(\mathrm{U}_{31}-\mathrm{U}_{11} \mathrm{U}_{33} / \mathrm{U}_{13}\right)^{\mathrm{m}}, \pi_{2}=\left(\mathrm{U}_{32}-\mathrm{U}_{12} \mathrm{U}_{33} / \mathrm{U}_{13}\right)^{\mathrm{m}}, \pi_{3}=\left(\mathrm{U}_{41}-\mathrm{U}_{43} \mathrm{U}_{11} / \mathrm{U}_{13}\right)^{\mathrm{m}}, \\
& \pi_{4}=\left(\mathrm{U}_{42}-\mathrm{U}_{43} \mathrm{U}_{12} / \mathrm{U}_{13}\right)^{\mathrm{m}}, \pi_{5}=\left(\pi_{1} \mathrm{U}_{46}-\pi_{3} \mathrm{U}_{36}\right)^{\mathrm{m}} /\left(\mathrm{U}_{44} \mathrm{U}_{36}-\mathrm{U}_{34} \mathrm{U}_{46}\right)^{\mathrm{m}}, \\
& \pi_{6}=\left(\pi_{2} \mathrm{U}_{46}-\pi_{4} \mathrm{U}_{36}\right)^{\mathrm{m}} /\left(\mathrm{U}_{44} \mathrm{U}_{36}-\mathrm{U}_{34} \mathrm{U}_{46}\right)^{\mathrm{m}}, \pi_{7}=\left(\pi_{1} \mathrm{U}_{44}-\pi_{3} \mathrm{U}_{34}\right)^{\mathrm{m}} /\left(\mathrm{U}_{46} \mathrm{U}_{34}-\mathrm{U}_{36} \mathrm{U}_{44}\right)^{\mathrm{m}}, \\
& \pi_{8}=\left(\pi_{2} \mathrm{U}_{44}-\pi_{4} \mathrm{U}_{34}\right)^{\mathrm{m}} /\left(\mathrm{U}_{46} \mathrm{U}_{34}-\mathrm{U}_{36} \mathrm{U}_{44}\right)^{\mathrm{m}}, \\
& \pi_{9}=\left(\pi_{5} \mathrm{U}_{64} \mathrm{U}_{77}-\pi_{5} \mathrm{U}_{74} \mathrm{U}_{64}+\pi_{7} \mathrm{U}_{66} \mathrm{U}_{77}-\pi_{7} \mathrm{U}_{76} \mathrm{U}_{67}\right)^{\mathrm{m}} /\left(\mathrm{U}_{75} \mathrm{U}_{67}-\mathrm{U}_{65} \mathrm{U}_{77}\right)^{\mathrm{m}}, \\
& \pi_{10}=\left(\pi_{6} \mathrm{U}_{64} \mathrm{U}_{77}-\pi_{6} \mathrm{U}_{74} \mathrm{U}_{64}+\pi_{8} \mathrm{U}_{66} \mathrm{U}_{77}-\pi_{8} \mathrm{U}_{76} \mathrm{U}_{67}\right)^{\mathrm{m}} /\left(\mathrm{U}_{75} \mathrm{U}_{67}-\mathrm{U}_{65} \mathrm{U}_{77}\right)^{\mathrm{m}}, \\
& \pi_{11}=\left(\pi_{5} \mathrm{U}_{64} \mathrm{U}_{75}-\pi_{5} \mathrm{U}_{74} \mathrm{U}_{65}+\pi_{7} \mathrm{U}_{66} \mathrm{U}_{75}-\pi_{7} \mathrm{U}_{76} \mathrm{U}_{65}\right)^{\mathrm{m}} /\left(\mathrm{U}_{65} \mathrm{U}_{77}-\mathrm{U}_{75} \mathrm{U}_{67}\right)^{\mathrm{m}}, \\
& \pi_{12}=\left(\pi_{6} \mathrm{U}_{64} \mathrm{U}_{75}-\pi_{6} \mathrm{U}_{74} \mathrm{U}_{65}+\pi_{8} \mathrm{U}_{66} \mathrm{U}_{75}-\pi_{8} \mathrm{U}_{76} \mathrm{U}_{65}\right)^{\mathrm{m}} /\left(\mathrm{U}_{65} \mathrm{U}_{77}-\mathrm{U}_{75} \mathrm{U}_{67}\right)^{\mathrm{m}} .
\end{aligned}
$$

Conflict of Interest: Authors declare that we do not have any conflict of interest.

Data Availability Statements: All data generated during this study are not applicable for sharing but are available on reasonable request as relevant data of system parameters are already accompanied with the figures.

Ethical Statement: The present research article does not contain any studies with human participants or animals by any of the authors. 


\section{References}

[1] Hong, G. Cai, J., Yang, S. X.: Dynamic analysis of a flexible hub-beam system with tip mass. Mechanics Research Communications, 32, 173-190 (2005)

[2] Chalhoub, N. G., Kfoury, G. A: Development of a robust nonlinear observer for a singlelink flexible manipulator. Nonlinear Dynamics, 39, 217-233 (2005)

[3] Saad, M., Akhrif, O., Saydy, L.: Analytical model of one flexible link system with nonlinear kinematics. Journal of Vibration and Control, 19(12), 1795-1806 (2012)

[4] Meng, Q., Lai, X., Wang, Y., Wu, M.: A fast stable control strategy based on system energy for a planar single-link flexible manipulator. Nonlinear Dynamics, published online, 02 June (2018)

[5] Fung, R, F., Chang, H. C.: Dynamic modelling of a non-linearly constrained flexible manipulator with a tip mass by Hamilton's principle. Journal of Sound and Vibration, 216(5), 751-769 (1998)

[6] Pratiher, B., Dwivedy, S. K.: Non-linear vibration of a single link viscoelastic Cartesian manipulator. International Journal of Non-Linear Mechanics, 43, 683-696 (2008)

[7] Qiu, Z.: Adaptive nonlinear vibration control of a Cartesian flexible manipulator driven by a ballscrew mechanism. Mechanical Systems and Signal Processing, Vol. 30, 248-266, (2012)

[8] Liu, Y., Li, W., Yang, X., Fan, M., Wang, Y., Lu, E.: Vibration response and power flow characteristics of a flexible manipulator with a moving base. Shock and Vibration, Vol. 2015, Article ID 589507, 8 pages (2015) 
[9] Basher, H. A.: Modeling and simulation of flexible robot manipulator with a prismatic joint. Proceedings 2007 IEEE SoutheastCon, Richmond, VA, 2007, 255-260, (2017).

[10] Ju, J., Li, W., Fan, M., Wang, Y., Yang, X.: Nonlinear modelling and dynamic stability analysis of a flexible Cartesian robotic manipulator with base disturbance and terminal load. Mechanical Sciences, 8, 221-234 ( 2017)

[11] Wu, S., Tang, S., Huang, K.: Vibration attenuation of a two-link flexible arm carried by a translational stage. Journal of Vibration and Control, Vol. 24 (23), 5650-5664, (2018)

[12] Emam, S. A.: Dynamics of a flexible-hub geometrically nonlinear beam with a tip mass. Journal of Vibration and Control, 16(13), 1989 -2000 (2010)

[13] My, C. A., Bien, D. X., Le, C. H., Packianather, M.: An efficient finite element formulation of dynamics for a flexible robot with different type of joints. Mechanism and Machine Theory, Vol 134, 267-288 (2019)

[14] Kim, H., Choi, S., Thompson, B. S.: Compliant control of a two-link flexible manipulator featuring piezoelectric actuators. Mechanism and Machine Theory, Vol. 36 (3), 411-424, (2001)

[15] Kalyoncu, M.: Mathematical modelling and dynamic response of a multi-straight-line path tracing flexible robot manipulator with rotating-prismatic joint. Applied Mathematical Modelling, Vol. 32 (6), 1087-1098, (2008)

[16] Coleman, M. P.: Analysis and computation of the vibration spectrum of the Cartesian flexible manipulator. Journal of Sound and Vibration, Vol. 274 (1-2), 445-454, (2004)

[17] Zhang, X., Zhang, D., Chen, S., Hong, J.: Modal characteristics of a rotating flexible beam with a concentrated mass based on the absolute nodal coordinate formulation. Nonlinear Dynamics, 88, 61-77, (2017) 
[18] Kumar, P., Pratiher, B.: Nonlinear modeling and vibration analysis of a twolink flexible manipulator coupled with harmonically driven flexible joints. Mechanism and Machine Theory, Vol. 131, 278-299 (2019)

[19] Pratiher, B, Bhowmick, S.: Nonlinear dynamic analysis of a Cartesian manipulator carrying an end effector placed at an intermediate position. Nonlinear Dynamics, 69, 539$553,(2012)$

[20] Hamed, Y. S., Alharthi, M. R., AlKhathami, H. K.: Nonlinear vibration behavior and resonance of a Cartesian manipulator system carrying an intermediate end effector. Nonlinear Dynamics, 91, 1429-1442, (2018)

[21] Endo, K., Yabuno, H.: Swing-up control of a three-link underactuated manipulator by high-frequency horizontal excitation. Transactions of ASME, Journal of Computational, and Nonlinear Dynamics, 8(1), (2012)

[22] Abe, A.: Trajectory planning for residual vibration suppression of a two-link rigid-flexible manipulator considering large deformation. Mechanism and Machine Theory, Vol. 44, 9, 1627-1639, (2009)

[23] Lochan, K., Roy, B. K., Subudhi, B.: Robust tip trajectory synchronisation between assumed modes modelled two-link flexible manipulators using second-order PID terminal SMC. Robotics and Autonomous Systems, 97, 108-124 (2017)

[24] Kalyoncu, M.: Mathematical modelling and dynamic response of a multi-straight-line path tracing flexible robot manipulator with rotating-prismatic joint. Applied Mathematical Modelling, Vol. 32 (6), 1087-1098, (2008) 
[25] Karagulle, H., Malgaca, L., Dirilmis, M., Akdag, M., Yavuz, S.: Vibration control of a two-link flexible manipulator. Journal of Vibration and Control, Vol. 23(12), 2023 - 2034, (2015)

[26] Du, H., Lim, K., Liew, K. M.: A nonlinear finite element model for dynamics of flexible manipulators. Mechanism and Machine Theory, Vol. 31 (8), 1109-1119 (1996)

[27] Jonker, B.: A finite element dynamic analysis of flexible manipulators. International Journal of Robotics Research, Vol. 9 (4), 59-74 (1990)

[28] Cao, X. T., Li, Y. C.: Robust RBF neural network force/position control of time varying constrained flexible manipulator. Control and Decision, 22, 977-982, (2008)

[29] Green, A., Sasiadek, J. Z.: Dynamics and trajectory tracking control of a two-link robot manipulator. Journal of Vibration and Control, Vol. 10 (10), 1415-1440 (2004)

[30] Botsali, F. M., Tinkir, M., Kalyoncu, M., Onen, U.: Fuzzy logic trajectory control of flexible robot manipulator with rotating prismatic joint. The 2nd International Conference on Computer and Automation Engineering (ICCAE), Singapore, 35-39 (2010)

[31] Bolandi, H., Esmaeilzadeh, S. M.: Analytical modelling and nonlinear stain feedback control of a flexible robot arm. Automatic Control and Computer Sciences, 42 (5), 236-248, (2008)

[32] Demydyuk, M. V, Hoshovska, N. V.: Parametric optimization of the transport operations of a two-link manipulator. Journal of Mathematical Sciences, 238(2), 174-188, (2019)

[33] Khairudin, M., Mohamed, Z., Husain, A. R., Mamat, R.: Dynamic characterization of a two-link flexible manipulator: theory and experiments. Advances in Robotics Research, 1(1), 061-079, (2014) 